\title{
Can Lorentz invariance violation affect the sensitivity of deep underground neutrino experiment?
}

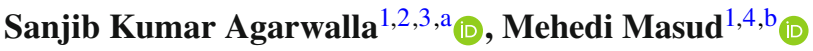 \\ ${ }^{1}$ Institute of Physics, Sachivalaya Marg, Sainik School Post, Bhubaneswar 751005, India \\ ${ }^{2}$ Homi Bhabha National Institute, Training School Complex, Anushakti Nagar, Mumbai 400085, India \\ ${ }^{3}$ International Centre for Theoretical Physics, Strada Costiera 11, 34151 Trieste, Italy \\ ${ }^{4}$ Astroparticle and High Energy Physics Group, Instituto de Física Corpuscular (CSIC/Universitat de València), Parc Cientific de Paterna. \\ C/Catedratico José Beltrán, 2, 46980 Paterna, València, Spain
}

Received: 15 February 2020 / Accepted: 30 July 2020 / Published online: 9 August 2020

(C) The Author(s) 2020

\begin{abstract}
We examine the impact of Lorentz Invariance Violation (LIV) in measuring the octant of $\theta_{23}$ and CP phases in the context of the Deep Underground Neutrino Experiment (DUNE). We consider the CPT-violating LIV parameters involving $e-\mu\left(a_{e \mu}\right)$ and $e-\tau\left(a_{e \tau}\right)$ flavors, which induce an additional interference term in neutrino and antineutrino appearance probabilities. This new interference term depends on both the standard CP phase $\delta$ and the new dynamical $\mathrm{CP}$ phase $\varphi_{e \mu} / \varphi_{e \tau}$, giving rise to new degeneracies among $\left(\theta_{23}\right.$, $\delta, \varphi)$. Taking one LIV parameter at-a-time and considering a small value of $\left|a_{e \mu}\right|=\left|a_{e \tau}\right|=5 \times 10^{-24} \mathrm{GeV}$, we find that the octant discovery potential of DUNE gets substantially deteriorated for unfavorable combinations of $\delta$ and $\varphi_{e \mu} / \varphi_{e \tau}$. The octant of $\theta_{23}$ can only be resolved at $3 \sigma$ if the true value of $\sin ^{2} \theta_{23} \lesssim 0.42$ or $\gtrsim 0.62$ for any choices of $\delta$ and $\varphi$. Interestingly, we also observe that when both the LIV parameters $a_{e \mu}$ and $a_{e \tau}$ are present together, they cancel out the impact of each other to a significant extent, allowing DUNE to largely regain its octant resolution capability. We also reconstruct the CP phases $\delta$ and $\varphi_{e \mu} / \varphi_{e \tau}$. The typical $1 \sigma$ uncertainty on $\delta$ is $10-15^{\circ}$ and the same on $\varphi_{e \mu} / \varphi_{e \tau}$ is $25-30^{\circ}$ depending on the choices of their true values.
\end{abstract}

\section{Introduction}

New opportunities have emerged on the neutrino oscillation frontier where almost all the available data can be nicely accommodated in a standard three-flavor oscillation framework [1], except for a few intriguing anomalies uncovered by the short-baseline experiments (for recent reviews see [2,3]). There are six fundamental parameters in the three-neutrino

\footnotetext{
a e-mail: sanjib@iopb.res.in (corresponding author)

be-mail:masud@ific.uv.es
}

(3v) mixing paradigm that govern the oscillation phenomena: (a) three leptonic mixing angles $\left(\theta_{12}, \theta_{13}, \theta_{23}\right)$, (b) one Dirac CP phase $(\delta)$, and c) two distinct mass-squared splittings ${ }^{1}\left(\Delta m_{21}^{2}, \Delta m_{32}^{2}\right)$. After establishing the phenomena of neutrino oscillation conclusively, neutrino physics has now entered into the precision era with an aim to address the following three fundamental pressing issues at unprecedented confidence level.

- Determining the value of charge-parity $(\mathrm{CP})$ violating phase $\delta$ - where establishing a value differing from both zero and $\pi$ would symbolize the discovery of CPviolation $(\mathrm{CPV})$ in the leptonic sector.

- Settling the pattern of neutrino masses. The present oscillation data cannot resolve whether $\Delta m_{31}^{2}\left(\equiv m_{3}^{2}-m_{1}^{2}\right)$ is positive or negative. It allows us to arrange the neutrino masses in two different fashions: $m_{3}>m_{2}>m_{1}$, called normal ordering (NO) where $\Delta m_{31}^{2}$ is positive and $m_{2}>m_{1}>m_{3}$, known as inverted ordering (IO) where $\Delta m_{31}^{2}$ is negative.

- Precise measurement of the mixing angle $\theta_{23}$. If it turns out to be non-maximal $\left(\theta_{23} \neq \pi / 4\right)$, then we can have two possibilities: $\theta_{23}$ can either lie in the lower octant (LO), i.e. $\theta_{23}<\pi / 4$ or in the higher octant (HO), i.e. $\theta_{23}>$ $\pi / 4$.

Presently running long-baseline neutrino oscillation experiments Tokai to Kamioka (T2K) [4] and NuMI Off-axis $v_{e}$ Appearance (NOvA) [5] have already started shading light on the above mentioned issues. Latest T2K results [6] hint

\footnotetext{
$\left.\overline{1 \Delta m_{21}^{2}\left(\equiv m_{2}^{2}\right.}-m_{1}^{2}\right)$ governs the oscillation in the solar sector and $\Delta m_{32}^{2}\left(\equiv m_{3}^{2}-m_{2}^{2}\right)$ is responsible for the oscillation in the atmospheric sector. Here, the neutrino mass eigenstate $m_{3}$ has the smallest electron component.
} 
towards a $\mathrm{HO}$ value for $\sin ^{2} \theta_{23}=0.53_{-0.04}^{+0.03}$ for both $\mathrm{NO}$ and IO. For the first time, T2K has been able to rule out a large range of values of $\delta$ around $\pi / 2$ at $3 \sigma$ C.L. irrespective of mass ordering. The $\mathrm{CP}$ conserving choices of $\delta$ (both 0 and $\pi$ ) are also excluded at $95 \%$ C.L. by the same data. The most recent measurements by the NOvA Collaboration [7] using both neutrinos and antineutrinos point towards NO, disfavoring IO at $1.9 \sigma$ C.L. and shows a weak preference for $\theta_{23}$ in $\mathrm{HO}$ over $\mathrm{LO}$ at a C.L. of $1.6 \sigma$. The NOvA data excludes most of the choices near $\delta=\pi / 2$ for IO at a C.L. $\geqslant 3 \sigma$. But these experiments still have a long way to go and hopefully, their results will be strengthened further with more statistics in near future. The global analyses of world neutrino data [8-11] also indicate towards NO at more than $3 \sigma$ C.L. and a non-maximal $\theta_{23}$ around $2 \sigma$ with a mild preference for HO. However, the value of the standard CP phase $\delta$ is still uncertain by a large extent.

The upcoming high-precision long-baseline neutrino oscillation experiments are expected to resolve these crucial issues at high confidence level and to provide a rigorous test of the three-flavor neutrino oscillation framework in the presence of Earth's matter effect [12-14]. These experiments include Deep Underground Neutrino Experiment (DUNE) [15,16], Tokai to Hyper-Kamiokande (T2HK) [17], Tokai to HyperKamiokande with a second detector in Korea (T2HKK) [18], and European Spallation Source $v$ Super Beam (ESSvSB) $[19,20]$. These facilities are supposed to measure the mixing angles and mass-squared differences with a precision below a few $\%$ and therefore, these next generation neutrino experiments may be sensitive to various Beyond the Standard Model (BSM) scenarios [21], which will complement the search for new physics at the ongoing LHC and future collider facilities. In this paper, we consider a specific BSM scenario of Lorentz Invariance Violation (LIV) [22,23] and analyze its impact on the measurements of $\theta_{23}$ octant and CPV at the DUNE facility.

The Standard Model (SM) is considered to be a lowenergy effective gauge theory of a more fundamental framework that also unifies gravitational interactions along with strong, weak, and electromagnetic interactions. The natural mass scale of that theory is governed by the Planck mass $\left(M_{P} \sim 10^{19} \mathrm{GeV}\right)$. There exist studies that propose spontaneous LIV and CPT violations ${ }^{2}$ in that more complete framework [25-29]. In the observable low-energy limit, this spontaneous violation of CPT/Lorentz symmetry can give rise to a minimal extension of the standard model through small perturbative terms suppressed by $M_{P}$. In the present work, we consider this minimal extension of the SM (as developed in [22,23,30-32]) which violates Lorentz invariance as well as CPT symmetry.

\footnotetext{
${ }^{2}$ In a seminal paper by $\mathrm{O}$. W. Greenberg, it was shown that CPT violation implies violation of Lorentz Invariance [24].
}

Neutrino experiments may determine the presence of Lorentz/CPT violation via the possible changes in neutrino oscillation probabilities, which can happen due to various reasons such as neutrino-antineutrino mixing, energy dependent effects on mass splittings, and time or direction dependent effects $[22,23,33,34]$. Several neutrino oscillation experiments such as liquid scintillator neutrino detector (LSND) [35], main injector neutrino oscillation search (MINOS) [36-38], mini booster neutrino experiment (MiniBooNE) [39], Double Chooz [40], Super-Kamiokande (SK) [41], IceCube [42,43], and T2K [44] have searched for these LIV/CPT-violating effects in their datasets and have placed competitive constraints on these LIV/CPT-violating parameters. Besides the above mentioned studies by the official Collaborations, there are also several other independent attempts on constraining LIV/CPT-violating parameters in the context of long-baseline accelerator neutrinos [45-51], shortbaseline reactor antineutrinos [52], atmospheric neutrinos [53-55], solar neutrinos [56], and high-energy astrophysical neutrinos [57-59]. Hadron colliders such as LHC can also provide unique opportunity to test LIV/CPT-violating effects at high energy $[60,61]$. A comprehensive list of the constraints on all the relevant LIV/CPT-violating parameters is available in Ref. [62]. In a recent work, the authors of [50] have performed a detailed analysis to put stronger bounds on the most relevant CPT-violating LIV parameters by simulating the upcoming DUNE experiment. Using these more tightly constrained CPT-violating LIV parameters, we study here for the first time, the octant sensitivity of DUNE in presence of the LIV parameters $\left(\left|a_{e \mu}\right|, \varphi_{e \mu}\right)$ and $\left(\left|a_{e \tau}\right|, \varphi_{e \tau}\right)$. Also, we have explored the capability of DUNE to reconstruct the true values of the standard Dirac CP phase $\delta$ and the LIV phases $\varphi_{e \mu}$ and $\varphi_{e \tau}$. Our analyses include the presence of both of these sets of LIV parameters individually as well as collectively. For recent status of searches of various BSM physics other than LIV using neutrino experiments, see $[2,3,63-69]$ and the references therein.

This paper is organized as follows. Section 2 gives a short overview of the theoretical background pertaining to LIV scenario and provides an analytical discussion on how LIV parameters alter the neutrino and antineutrino appearance probability expressions by introducing an additional interference term, which depends on both the standard CP phase $\delta$ and the new dynamical CP phase $\varphi_{e \mu} / \varphi_{e \tau}$, giving rise to new degeneracies among $\theta_{23}, \delta$, and $\varphi$. In the same section, we also derive approximate analytical expressions to show how these new degeneracies affect the measurement of octant of $\theta_{23}$. Section 3 discusses the important detector properties and the $\Delta \chi^{2}$ analysis procedure. In Sect. 4, we show how various LIV parameters affect the exact numerical transition probability $P_{\mu e}$. In the same section, we also give bi-event plots to depict how much variation one can expect in the neutrino and antineutrino appearance event rates due 
to various LIV parameters. We present our main results concerning the octant discovery potential and the capability of reconstruction of the CP phases in Sect. 5. Finally, in Sect. 6, we summarize our results and conclude. In Appendix A, we explore the octant discovery potential of DUNE assuming the presence of LIV in data, but not in fit (theory).

\section{LIV formalism}

Lorentz Invariance violating neutrinos and antineutrinos are effectively described by the Lagrangian density $[22,32]$,

$\mathcal{L}=\frac{1}{2} \bar{\psi}(i \not \partial-M+\hat{\mathcal{Q}}) \psi+h . c .$,

where, $\hat{\mathcal{Q}}$ is a generic Lorentz Invariance violating operator and the spinor $\psi$ describes the neutrino field. The first term on the right hand side (RHS) of Eq. 1 is the usual kinetic term, the second part involves the mass term with the mass matrix $\mathrm{M}$ and the 3rd term gives rise to the Lorentz Invariance violating effect, which is small and perturbative in nature, possibly arising from Planck-suppressed effects. Considering only the renormalizable Dirac couplings of the theory, we can start from the Lorentz Invariance violating Lagrangian [32],

$$
\begin{aligned}
\mathcal{L}_{\mathrm{LIV}}=- & \frac{1}{2}\left[a_{\alpha \beta}^{\mu} \bar{\psi}_{\alpha} \gamma_{\mu} \psi_{\beta}+b_{\alpha \beta}^{\mu} \bar{\psi}_{\alpha} \gamma_{5} \gamma_{\mu} \psi_{\beta}\right. \\
& \left.-i c_{\alpha \beta}^{\mu \nu} \bar{\psi}_{\alpha} \gamma_{\mu} \partial_{\nu} \psi_{\beta}-i d_{\alpha \beta}^{\mu \nu} \bar{\psi}_{\alpha} \gamma_{5} \gamma_{\mu} \partial_{\nu} \psi_{\beta}\right]+ \text { h.c. }
\end{aligned}
$$

The observable effect on the left handed neutrinos is controlled by the combinations

$\left(a_{L}\right)_{\alpha \beta}^{\mu}=(a+b)_{\alpha \beta}^{\mu}, \quad\left(c_{L}\right)_{\alpha \beta}^{\mu \nu}=(c+d)_{\alpha \beta}^{\mu \nu}$,

which are constant hermitian matrices in the flavor space that can modify the standard vacuum Hamiltonian. The first combination is relevant for CPT-violating neutrinos, whereas the second combination is only relevant for CPTeven Lorentz-violating neutrinos. In this work, we will focus on the isotropic component of the Lorentz-violating terms and therefore, we will fix the $(\mu, v)$ indices to zero $(0)$. To simplify our notation, from now on, we will denote ${ }^{3}$ the parameter $\left(a_{L}\right)_{\alpha \beta}^{0}$ as $a_{\alpha \beta}$ and $\left(c_{L}\right)_{\alpha \beta}^{00}$ as $c_{\alpha \beta}$.

Explicitly, one can write the Lorentz-violating contribution to the full oscillation Hamiltonian

$H=H_{\mathrm{vac}}+H_{\mathrm{mat}}+H_{\mathrm{LIV}}$

\footnotetext{
3 These components are defined in the Sun-centered celestial equatorial frame [22].
}

such that,

$$
\begin{aligned}
H_{\mathrm{vac}}= & \frac{1}{2 E} U\left(\begin{array}{ccc}
m_{1}^{2} & 0 & 0 \\
0 & m_{2}^{2} & 0 \\
0 & 0 & m_{3}^{2}
\end{array}\right) U^{\dagger} ; \\
H_{\mathrm{mat}}= & \sqrt{2} G_{F} N_{e}\left(\begin{array}{cc}
1 & \\
& 0 \\
& 0
\end{array}\right) ; \\
H_{\mathrm{LIV}}= & \left(\begin{array}{lll}
a_{e e} & a_{e \mu} & a_{e \tau} \\
a_{e \mu}^{*} & a_{\mu \mu} & a_{\mu \tau} \\
a_{e \tau}^{*} & a_{\mu \tau}^{*} & a_{\tau \tau}
\end{array}\right) \\
& -\frac{4}{3} E\left(\begin{array}{lll}
c_{e e} & c_{e \mu} & c_{e \tau} \\
c_{e \mu}^{*} & c_{\mu \mu} & c_{\mu \tau} \\
c_{e \tau}^{*} & c_{\mu \tau}^{*} & c_{\tau \tau}
\end{array}\right),
\end{aligned}
$$

where, $U$ is the neutrino mixing matrix, $m_{i}$ 's are the neutrino mass eigenstates, $G_{F}$ is the Fermi coupling constant, and $N_{e}$ is the electron density along the neutrino trajectory. The $a_{\alpha \beta}$ 's and $c_{\alpha \beta}$ 's are the LIV parameters. In Eq. 6 , the factor $-4 / 3$ in front of the second term arises from the non-observability of the Minkowski trace of $c_{L}$, which forces the components $x x, y y$, and $z z$ to be related to the 00 component [22]. In this work, we consider the presence of Lorentz-violating effects only due to the first type of terms ${ }^{4}$ in Eq. 6. Updated constraints on $a_{\alpha \beta}$ 's, mainly from Super-Kamiokande, can be found in Refs. [41,62]. Note that, after considering only the CPT-violating LIV terms ( $a_{\alpha \beta}$ 's), the LIV effect looks similar to the effect of neutral current (NC) non-standard interaction (NSI) during neutrino propagation, which can be described in the following fashion

$H^{\prime}=H_{\mathrm{vac}}+H_{\mathrm{mat}}+H_{\mathrm{NSI}}$,

where the NSI term is parameterized as

$H_{\mathrm{NSI}}=\sqrt{2} G_{F} N_{e}\left(\begin{array}{ccc}\varepsilon_{e e}^{m} & \varepsilon_{e \mu}^{m} & \varepsilon_{e \tau}^{m} \\ \varepsilon_{\mu e}^{m} & \varepsilon_{\mu \mu}^{m} & \varepsilon_{\mu \tau}^{m} \\ \varepsilon_{\tau e}^{m} & \varepsilon_{\tau \mu}^{m} & \varepsilon_{\tau \tau}^{m}\end{array}\right)$.

Here, $N_{e}$ corresponds to the electron number density along the neutrino trajectory and the parameters $\varepsilon_{\alpha \beta}^{m}$ denote the strength of the NSI. One thus finds a correlation between the NSI and LIV scenario through the following relation [70],

$\varepsilon_{\alpha \beta}^{m} \equiv \frac{a_{\alpha \beta}}{\sqrt{2} G_{F} N_{e}}$.

However, there are important differences between these two scenarios $[70,71]$. NSI during neutrino propagation is basically an exotic matter effect and hence, plays no role in vacuum, whereas the type of LIV considered here is an intrinsic effect, present even in vacuum. Nevertheless, the equivalence

\footnotetext{
4 Therefore, we can argue that the LIV scenario which we analyze in the present work is also CPT-violating in nature.
} 
in Eq. 9 allows the study of the LIV parameters in longbaseline experiments following an approach, which is quite similar to the treatment of NSI in neutrino propagation.

In this paper, we only consider the LIV parameters $a_{e \mu}$ ( $\left.\equiv\left|a_{e \mu}\right| e^{i \varphi_{e \mu}}\right)$ and $a_{e \tau}\left(\equiv\left|a_{e \tau}\right| e^{i \varphi_{e \tau}}\right)$ since these parameters influence the most $v_{\mu} \rightarrow v_{e}$ appearance channel, which drives the CPV and octant sensitivity in a typical longbaseline experiment such as DUNE. The probability expression for $v_{\mu} \rightarrow v_{e}$ oscillation channel in presence of the LIV parameters $a_{e \mu}$ and $a_{e \tau}$ can be written as (following the similar expressions in presence of the NSI parameters $\varepsilon_{e \mu}$ and $\varepsilon_{e \tau}$ in Refs. [72-74]):

$P_{\mu e} \simeq P_{\mu e}(\mathrm{SI})+P_{\mu e}\left(a_{e \mu}\right)+P_{\mu e}\left(a_{e \tau}\right)$,

where, the three terms on the RHS are described below. The first term originating from the standard interaction (SI) of neutrinos with the Earth's matter is given by

$P_{\mu e}(\mathrm{SI}) \simeq X+Y \cos (\delta+\Delta)$,

where,

$$
\begin{gathered}
X=4 s_{13}^{2} c_{13}^{2} s_{23}^{2} \frac{\sin ^{2}[(1-\hat{A}) \Delta]}{(1-\hat{A})^{2}} ; \\
Y=8 \alpha s_{12} c_{12} s_{23} c_{23} s_{13} c_{13} \frac{\sin \hat{A} \Delta}{\hat{A}} \frac{\sin [(1-\hat{A}) \Delta]}{1-\hat{A}}, \\
\hat{A}=\frac{2 \sqrt{2} G_{F} N_{e} E}{\Delta m_{31}^{2}} ; \quad \Delta=\frac{\Delta m_{31}^{2} L}{4 E} ; \\
s_{i j}=\sin \theta_{i j} ; \quad c_{i j}=\cos \theta_{i j} ; \quad \alpha=\frac{\Delta m_{21}^{2}}{\Delta m_{31}^{2}} .
\end{gathered}
$$

In writing the expression for $P_{\mu e}(\mathrm{SI})$ in Eq. 11, we neglect the solar term $\alpha^{2} \sin ^{2} 2 \theta_{12} c_{23}^{2} \frac{\sin ^{2} \hat{A} \Delta}{\hat{A}^{2}}$. This is due to the fact that by considering the values of the oscillation parameters as $\theta_{12}=34.5^{\circ}, \theta_{13}=8.45^{\circ}, \theta_{23}=47.7^{\circ}, \Delta m_{21}^{2}=7.5 \times$ $10^{-5} \mathrm{eV}^{2}, \Delta m_{31}^{2}=2.5 \times 10^{-3} \mathrm{eV}^{2}$ (which are in agreement with [8-11]), we find that the solar term, being proportional to $\alpha^{2}$, is roughly suppressed by 3-4 orders of magnitude as compared to the other two terms as shown in Eq. 11.

To describe the second and the third terms of the RHS of Eq. 10, describing the effect of LIV due to the presence of $a_{e \mu}$ and $a_{e \tau}$ respectively, we take the similar approach as followed in the context of NC NSI in [72-74] with the NSI parameter $\varepsilon_{\alpha \beta}$ replaced appropriately (as in Eq. 9). Thus, the LIV terms in Eq. 10 can be written in the following compact form:

$$
\begin{aligned}
P_{\mu e}\left(a_{e \beta}\right) \simeq & \frac{4\left|a_{e \beta}\right| \hat{A} \Delta s_{13} \sin 2 \theta_{23} \sin \Delta}{\sqrt{2} G_{F} N_{e}} \\
& \times\left[Z_{e \beta} \sin \left(\delta+\varphi_{e \beta}\right)+W_{e \beta} \cos \left(\delta+\varphi_{e \beta}\right)\right], \quad(\beta=\mu, \tau)
\end{aligned}
$$

where,

$$
\begin{aligned}
& Z_{e \beta}= \begin{cases}-c_{23} \sin \Delta, & \text { if } \beta=\mu . \\
s_{23} \sin \Delta, & \text { if } \beta=\tau .\end{cases} \\
& W_{e \beta}= \begin{cases}c_{23}\left(\frac{s_{23}^{2} \sin \Delta}{c_{23}^{2} \Delta}+\cos \Delta\right), & \text { if } \beta=\mu . \\
s_{23}\left(\frac{\sin \Delta}{\Delta}-\cos \Delta\right), & \text { if } \beta=\tau .\end{cases}
\end{aligned}
$$

We note that the factor $\frac{\hat{A} \Delta}{\sqrt{2} G_{F} N_{e}}$ in Eq. 13 becomes $L / 2$ (see Eq. 12.), thereby making the LIV effect considered here explicitly matter independent.

Following [73], now we explain the issue of octant sensitivity of $\theta_{23}$ by expressing the atmospheric mixing angle as:

$\theta_{23}=\frac{\pi}{4} \pm \eta$,

such that the positive angle $\eta$ quantifies the deviation from the maximal mixing. The positive (negative) sign corresponds to $\mathrm{HO}$ (LO). The current three-flavor global analyses [8-11] indicate that $\theta_{23}$ cannot deviate from $45^{\circ}$ by more than $\sim 6^{\circ}$, i.e., $\sin ^{2} \theta_{23}$ must be in the range $[0.4,0.6]$. Therefore, one has $\eta \lesssim 0.1$, and we can use the expansions,

$s_{23}^{2} \simeq \frac{1}{2} \pm \eta ; \quad c_{23}^{2} \simeq \frac{1}{2} \mp \eta ; \quad \sin 2 \theta_{23} \simeq 1$.

An experiment is sensitive to the octant of $\theta_{23}$ if at the probability level the following difference between the true octant (tr) and test octant (ts) is nonzero at a detectable level.

$\Delta P=P_{\mu e}^{\mathrm{tr}}\left(\theta_{23}^{\mathrm{tr}}, \delta^{\mathrm{tr}}, \varphi^{\mathrm{tr}}\right)-P_{\mu e}^{\mathrm{ts}}\left(\theta_{23}^{\mathrm{ts}}, \delta^{\mathrm{ts}}, \varphi^{\mathrm{ts}}\right)$.

Since $P_{\mu e}$ consists of the three terms in Eq. 10, we can write,

$\Delta P \simeq \Delta P_{\mu e}(\mathrm{SI})+\Delta P_{\mu e}\left(a_{e \mu}\right)+\Delta P_{\mu e}\left(a_{e \tau}\right)$.

To analyse the three terms on the RHS of Eq. 18, we take the case of $\mathrm{HO}(\mathrm{LO})$ as true (test) octant as an example. Then for the SI term,

$\Delta P_{\mu e}(\mathrm{SI})=\Delta X+\Delta Y\left[\cos \left(\delta^{\mathrm{HO}}+\Delta\right)-\cos \left(\delta^{\mathrm{LO}}+\Delta\right)\right]$,

where,

$$
\begin{aligned}
& \Delta X \simeq 8 \eta s_{13}^{2} c_{13}^{2} \frac{\sin ^{2}[(1-\hat{A}) \Delta]}{(1-\hat{A})^{2}} ; \\
& \Delta Y \simeq 4 \alpha s_{12} c_{12} s_{13} c_{13} \frac{\sin \hat{A} \Delta}{\hat{A}} \frac{\sin [(1-\hat{A}) \Delta]}{1-\hat{A}} .
\end{aligned}
$$

The LIV contribution to Eq. 18 can be written as, 


$$
\begin{aligned}
\Delta P_{\mu e}\left(a_{e \beta}\right) \simeq & \frac{4\left|a_{e \beta}\right| \hat{A} \Delta s_{13} \sin 2 \theta_{23} \sin \Delta}{\sqrt{2} G_{F} N_{e}} \\
& \times\left[\Delta Z_{e \beta}\left\{\sin \left(\delta^{\mathrm{HO}}+\varphi_{e \beta}^{\mathrm{HO}}\right)-\sin \left(\delta^{\mathrm{LO}}+\varphi_{e \beta}^{\mathrm{LO}}\right)\right\}\right. \\
& \left.+\Delta W_{e \beta}\left\{\cos \left(\delta^{\mathrm{HO}}+\varphi_{e \beta}^{\mathrm{HO}}\right)-\cos \left(\delta^{\mathrm{LO}}+\varphi_{e \beta}^{\mathrm{LO}}\right)\right\}\right],
\end{aligned}
$$

where,

$$
\Delta Z_{e \beta} \simeq \mp \frac{1}{\sqrt{2}} \sin \Delta,
$$

[where the $-(+) \operatorname{sign}$ is for $\beta=\mu(\tau)$ ],

$\Delta W_{e \beta} \simeq \begin{cases}\frac{1}{\sqrt{2}}\left[\frac{\sin \Delta}{\Delta}+\cos \Delta\right], & \text { if } \beta=\mu, \\ \frac{1}{\sqrt{2}}\left[\frac{\sin \Delta}{\Delta}-\cos \Delta\right], & \text { if } \beta=\tau .\end{cases}$

In DUNE, neutrinos and antineutrinos travel the distance of $L=1300 \mathrm{~km}$ and for this baseline, the line-averaged constant Earth matter density turns out to be $\rho=2.95 \mathrm{~g} / \mathrm{cm}^{3}$ [75]. We also assume that Earth's matter is electrically neutral and isoscalar for which we have $N_{e}=N_{p}=N_{n}$, where $N_{p}$, $N_{n}$ are the proton and neutron densities respectively. Under this assumption, the relative number density $Y_{e}\left(\equiv \frac{N_{e}}{N_{p}+N_{n}}\right)$ comes out to be 0.5 . Also note that for DUNE baseline, the first oscillation maximum ( $\Delta \simeq \pi / 2$ ) occurs at $E \approx 2.5 \mathrm{GeV}$ assuming $\Delta m_{31}^{2}=2.5 \times 10^{-3} \mathrm{eV}^{2}$. With these benchmark choices of parameters, we obtain the following approximate numerical values:

$$
\begin{aligned}
& \Delta \simeq \pi / 2, \\
& \sqrt{2} G_{F} N_{e} \simeq\left[7.6 \times Y_{e} \times 10^{-14} \times \rho\left(\mathrm{g} / \mathrm{cm}^{3}\right)\right] \mathrm{eV} \\
& \simeq 1.12 \times 10^{-13} \mathrm{eV}, \\
& \hat{A}=\frac{2 \sqrt{2} G_{F} N_{e} E}{\Delta m_{31}^{2}} \simeq 0.23, \\
& \frac{\sin (1-\hat{A}) \Delta}{1-\hat{A}} \simeq 1.21, \\
& \frac{\sin \hat{A} \Delta}{\hat{A}} \simeq 1.54 .
\end{aligned}
$$

Now, to have an idea about the magnitude of the coefficients in Eqs. 19 and 21, we use the values of the oscillation parameters mentioned before and also Eqs. 20, 22, 23, and obtain the following at the 1 st oscillation maxima:

$$
\begin{aligned}
\Delta P_{\mu e}(\mathrm{SI}) \simeq & \frac{\eta}{0.05} 1.26 \times 10^{-2}+1.5 \\
& \times 10^{-2}\left[\cos \left(\delta^{\mathrm{HO}}+\Delta\right)-\cos \left(\delta^{\mathrm{LO}}+\Delta\right)\right],
\end{aligned}
$$

$$
\begin{aligned}
\Delta P_{\mu e}\left(a_{e \beta}\right) \simeq & {\left[\frac{\left|a_{e \beta}\right| \mathrm{GeV}^{-1}}{5 \times 10^{-24}}\right]\left[\mp 0.67 \times 10^{-2}\right.} \\
& \times\left\{\sin \left(\delta^{\mathrm{HO}}+\varphi_{e \beta}^{\mathrm{HO}}\right)-\sin \left(\delta^{\mathrm{LO}}+\varphi_{e \beta}^{\mathrm{LO}}\right)\right\} \\
& +0.42 \times 10^{-2}\left\{\cos \left(\delta^{\mathrm{HO}}+\varphi_{e \beta}^{\mathrm{HO}}\right)\right. \\
& \left.\left.-\cos \left(\delta^{\mathrm{LO}}+\varphi_{e \beta}^{\mathrm{LO}}\right)\right\}\right]
\end{aligned}
$$

where, $-(+)$ sign is for $\beta=\mu(\tau)$. It is clear from Eqs. 24 and 25 that for $\left|a_{e \mu}\right|\left(\left|a_{e \tau}\right|\right) \gtrsim 10^{-24} \mathrm{GeV}$, $\Delta P_{\mu e}\left(a_{e \mu}\right)\left(\Delta P_{\mu e}\left(a_{e \tau}\right)\right)$ becomes comparable to the standard interference term in $\Delta P_{\mu e}(\mathrm{SI})$. Moreover, $\Delta P_{\mu e}\left(a_{e \mu}\right)$ and $\Delta P_{\mu e}\left(a_{e \tau}\right)$ depend not only on the standard CP phase $\delta$, but also on the new dynamical $\mathrm{CP}$ phase $\varphi_{e \mu} / \varphi_{e \tau}$ related to the LIV. Due to this extra degree of freedom in $\Delta P_{\mu e}\left(a_{e \mu}\right) / \Delta P_{\mu e}\left(a_{e \tau}\right)$, the octant sensitivity can potentially become worse for unfavorable combinations of $\delta$ and $\varphi_{e \mu / e \tau}$. In addition, we note that the first terms in $\Delta P_{\mu e}\left(a_{e \mu}\right)$ and $\Delta P_{\mu e}\left(a_{e \tau}\right)$ appear with the opposite sign. It suggests that when both the LIV parameters $a_{e \mu}$ and $a_{e \tau}$ are present together, their effect may get cancelled to a large extent, and the chances of measuring octant in DUNE remain intact.

\section{Simulation details}

The proposed deep underground neutrino experiment (DUNE) is a world-class facility which is going to unravel some fundamental issues in neutrino sector, namely, the measurement of leptonic CP-violation, the determination of the neutrino mass ordering, and the precision measurement of the neutrino mixing parameters $[15,16,76,77]$. In order to simulate DUNE, we use the GLoBES package $[78,79]$ with the most recent DUNE configuration files provided by the collaboration [80]. To analyze the Lorentz-violating scenario, we perform our simulation of the DUNE experiment using the GLoBES-extension snu.c as described in Refs. [81,82]. This extension was originally introduced in GLoBES software to study non-standard neutrino interactions and sterile neutrinos in the context of long-baseline experiments. For the present analysis, we modify the definition of the neutrino oscillation probability function inside snu.c by implementing the Lorentz-violating Hamiltonian as given in Eq. 6. We assume a total run-time of 7 years with 3.5 years in the neutrino mode and the remaining 3.5 years in the antineutrino mode with an on-axis 40 kiloton liquid argon far detector (FD) housed at the Homestake Mine in South Dakota over a baseline of $1300 \mathrm{~km}$. The optimized neutrino beam is obtained from a G4LBNF simulation $[83,84]$ of the LBNF beam line using NuMI-style focusing. The neutrino beam is generated using $80 \mathrm{GeV}$ proton beam having a beam power of $1.07 \mathrm{MW}$, 
which can deliver $1.47 \times 10^{21}$ protons on target per calendar year. It corresponds to a total exposure of $300 \mathrm{kt} \cdot \mathrm{MW} \cdot$ years.

To simulate the DUNE event spectra, we consider the reconstructed neutrino and antineutrino energy range of 0 to $20 \mathrm{GeV}$ for both appearance and disappearance channels. While preparing our sensitivity plots, we perform a full spectral analysis with total 71 bins in the entire energy range having non-uniform bin widths. We have total 64 bins each having a width of $0.125 \mathrm{GeV}$ in the energy range of $0-8$ $\mathrm{GeV}$ and 7 bins with variable widths beyond $8 \mathrm{GeV}$ [80]. While estimating the signal and background event rates in the appearance and disappearance modes, we properly take into account the "wrong-sign" components, which are present in the beam. We do so for both $v_{e} / \bar{v}_{e}$ and $v_{\mu} / \bar{v}_{\mu}$ candidate events. We calculate the full three-flavour neutrino oscillation probabilities in matter considering the line-averaged constant Earth matter density of $2.95 \mathrm{~g} / \mathrm{cm}^{3}$ following the standard Preliminary Reference Earth Model (PREM) [85].

The main sources of backgrounds for the appearance events in neutrino and antineutrino modes are the intrinsic $v_{e} / \bar{v}_{e}$ contamination in the beam, the $\mu^{-} / \mu^{+}$events which are misidentified as $e^{-} / e^{+}$events, backgrounds arising from $v_{\tau} / \bar{v}_{\tau}$ appearance, and the NC events. For the disappearance events in neutrino and antineutrino modes, the main backgrounds stem from the $\mathrm{NC}$ events and $v_{\tau} / \bar{v}_{\tau}$ appearance. We incorporate the systematic uncertainties following Ref. [80]. We consider an independent normalization uncertainty of $2 \%$ on both $v_{e}$ and $\bar{v}_{e}$ signal modes, while the $v_{\mu}$ and $\bar{v}_{\mu}$ signal modes have uncorrelated normalization errors of $5 \%$. As far as the normalization uncertainties on various backgrounds are concerned, they vary in the range of 5-20\% with possible correlations among various sources of backgrounds.

To obtain the sensitivity results, we numerically calculate the $\Delta \chi^{2}$ between the true and test event spectra using GLoBES. Unless mentioned otherwise, we consider three different benchmark values of $\left|a_{e \beta}\right|=10^{-24} \mathrm{GeV}, 5 \times 10^{-24}$ $\mathrm{GeV}$, and $10^{-23} \mathrm{GeV}$ (where $\beta$ can be $\mu$ or $\tau$ ) while generating the true event spectra. While showing our results in Sect. 5, we always marginalize over the test $\left|a_{e \beta}\right|$ (where $\beta$ can be $\mu$ or $\tau$ ) in the range of $5 \times 10^{-25} \mathrm{GeV}$ to $5 \times 10^{-23}$ $\mathrm{GeV}$ in the fit. For a true value of $\delta$ and $\varphi_{e \beta}$, the true number of events in the $i$-th energy bin $N_{i}\left(\theta_{23}^{\text {true }}, \delta^{\text {true }}, \varphi_{e \beta}^{\text {true }}\right)$ is estimated by assuming a true octant scenario, which can be either true lower octant (i.e., $\theta_{23}^{\text {true }}<\pi / 4$ ) or true higher octant (i.e., $\left.\theta_{23}^{\text {true }}>\pi / 4\right)$. The fixed true and test values of the solar oscillation parameters and $1-3$ mixing angle are $\theta_{12}=34.5^{\circ}$, $\Delta m_{21}^{2}=7.5 \times 10^{-5} \mathrm{eV}^{2}$, and $\theta_{13}=8.45^{\circ}$. As far as the atmospheric mass-squared difference is concerned, we generate the data with a true value of $\Delta m_{31}^{2}=2.5 \times 10^{-3} \mathrm{eV}^{2}$ and we marginalize over test $\Delta m_{31}^{2}$ in the fit in its present $3 \sigma$ allowed range of $(2.41-2.6) \times 10^{-3} \mathrm{eV}^{2}$. We assume
NO both in data and theory. ${ }^{5}$ The theoretical event spectra are generated assuming the opposite/wrong octant scenario, where $\theta_{23}^{\text {test }}$ is marginalized over all possible values in the wrong octant only. $\delta^{\text {test }}$ and $\varphi_{e \beta}^{\text {test }}$ are marginalized over the full parameter space of $[-\pi, \pi] .\left|a_{e \beta}\right|^{\text {test }}$ is marginalized in the range of $5 \times 10^{-25} \mathrm{GeV}$ to $5 \times 10^{-23} \mathrm{GeV}$ as mentioned above. The $\Delta \chi^{2}$ thus gives a quantitative idea about the capability of the experiment to distinguish the true octant scenario from the wrong octant. ${ }^{6}$

\section{Transition probability and bi-event plots}

To demonstrate the impact of LIV, we have plotted $P_{\mu e}$ as a function of energy in Fig. 1 for both SI (black curves) and in presence of LIV (red curves). The left (right) panels assume the presence of the single LIV parameter $a_{e \mu}\left(a_{e \tau}\right)$. The top panels refer to representative $\mathrm{CP}$ conserving values $[0,0]$ of the two relevant phases $\left[\delta, \varphi_{e \beta}\right]$ as indicated in each panel. The bottom panels are for representative maximal CP-violating choices $[-\pi / 2,-\pi / 2]$ of the two CP phases $\left[\delta, \varphi_{e \beta}\right]$ as mentioned in each panel. For the purpose of illustration, here, we consider a relatively large strength of the LIV parameter $\left(\left|a_{e \mu}\right|\right.$ or $\left|a_{e \tau}\right|$ is taken to be $\left.2 \times 10^{-23} \mathrm{GeV}\right)$. As far as the three-flavor oscillation parameters are concerned, we consider the values $\theta_{12}=34.5^{\circ}, \theta_{13}=8.45^{\circ}, \theta_{23}=$ $47.7^{\circ}, \Delta m_{21}^{2}=7.5 \times 10^{-5} \mathrm{eV}^{2}, \Delta m_{31}^{2}=2.5 \times 10^{-3} \mathrm{eV}^{2}$, and assume NO. Figure 1 clearly demonstrates that the modifications in $v_{\mu} \rightarrow v_{e}$ transition probability due to the presence of LIV parameters depend upon the values of the CP phases $\left(\delta, \varphi_{e \mu / e \tau}\right)$. The excellent energy resolution in DUNE may enable us to study the changes in the reconstructed event spectra due to different choices of the CP phases $\left(\delta, \varphi_{e \mu / e \tau}\right)$, which in turn, may help us to reconstruct the values of these $\mathrm{CP}$ phases with reasonable accuracy.

Now, we introduce the bi-event plots (see Fig. 2) in which the $\mathrm{x}$-axis (y-axis) denotes the total number of appearance events in neutrino (antineutrino) mode. In all the panels, the solid/dashed ellipses depict the SI case, while the colored blobs portray the SI+LIV scheme. The ellipses are obtained by varying the standard Dirac $\mathrm{CP}$ phase $\delta$ in the range $[-\pi, \pi]$. In case of SI+LIV, there are more than one $\mathrm{CP}$ phase and due to the simultaneous variation of these phases in their allowed ranges, we have a convolution of an infinite

\footnotetext{
5 Since the sensitivity of DUNE to exclude the wrong mass ordering is very high [77], we keep the ordering same in both true and test datasets while performing our simulation. We have checked that DUNE can discriminate between NO and IO at high confidence level even in presence of the LIV parameters. It becomes possible due to the crucial spectral information provided by the on-axis wide-band muon-(anti)neutrino beam in DUNE.

6 The $\Delta \chi^{2}$ is calculated using the method of pull [86-89]. Also, this $\Delta \chi^{2}$ is valid in the frequentist method of hypotheses testing $[87,90]$.
} 
Fig. $1 v_{\mu} \rightarrow v_{e}$ transition probability as a function of neutrino energy. In each panel, black curve shows the probability considering only the standard interaction (SI). The red curves depict how the LIV parameters $a_{e \mu}$ (left panels) and $a_{e \tau}$ (right panels) affect $P_{\mu e}$.

The strength of the LIV parameters is assumed to be $\left|a_{e \mu}\right|=\left|a_{e \tau}\right|=2 \times 10^{-23} \mathrm{GeV}$ (taken one at-a-time). In top (bottom) panels, we assume $\mathrm{CP}$-conserving (CP-violating) values of the two relevant phases $\delta$ and $\varphi_{e \beta}$, whose values are mentioned in each panel. Here, we assume NO and for the three-flavor oscillation parameters, we take the values $\theta_{12}=34.5^{\circ}, \theta_{13}=8.45^{\circ}, \theta_{23}=$ $47.7^{\circ}, \Delta m_{21}^{2}=7.5 \times 10^{-5} \mathrm{eV}^{2}$, and $\Delta m_{31}^{2}=2.5 \times 10^{-3} \mathrm{eV}^{2}$

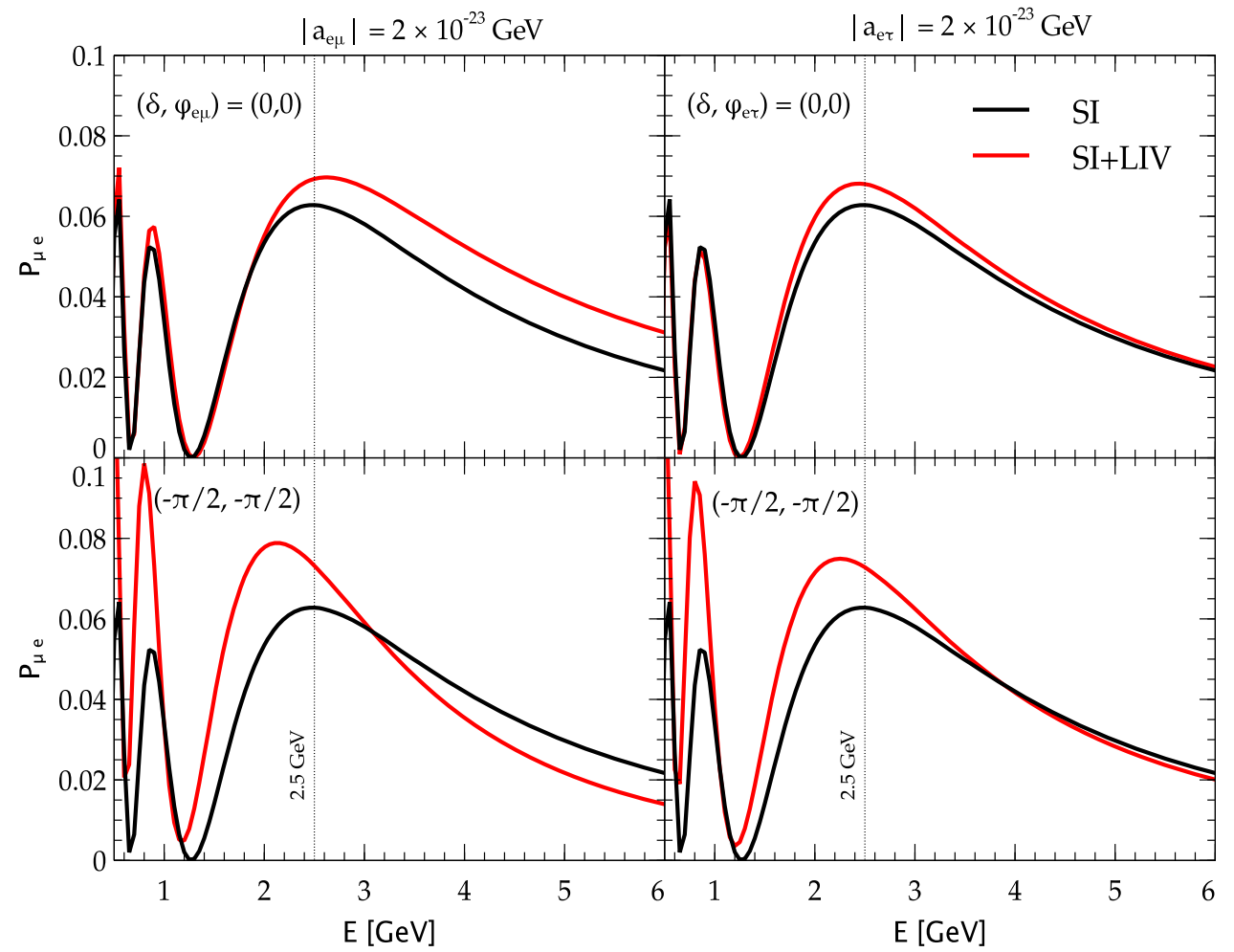

ensemble of ellipses with different orientations, which give rise to the colored blobs, In left (middle) panel, we obtain the blobs by varying the $\mathrm{CP}$ phases $\delta, \varphi_{e \mu}\left(\delta, \varphi_{e \tau}\right)$. In right panel, we vary three CP phases $\delta, \varphi_{e \mu}$, and $\varphi_{e \tau}$ at the same time in their allowed ranges of $[-\pi, \pi]$. In all the panels, the strength of the LIV parameter is taken to be $5 \times 10^{-24} \mathrm{GeV}$, which is consistent with [50]. For both SI and SI+LIV, we study four possible cases: two possible mass orderings (NO and IO) together with two possible octants (LO and HO), as mentioned in the figure legends. The black solid (dashed) ellipse corresponds to the NO-LO (IO-LO) case, while the red solid (dashed) ellipse represents the NO-HO (IO-HO) scenario. In case of $\mathrm{NO}$, we take the values of the oscillation parameters as $\theta_{12}=34.5^{\circ}, \Delta m_{21}^{2}=7.5 \times 10^{-5} \mathrm{eV}^{2}, \theta_{13}=$ $8.45^{\circ}, \Delta m_{31}^{2}=2.5 \times 10^{-3} \mathrm{eV}^{2}$, and $\theta_{23}=42.3^{\circ}\left(47.7^{\circ}\right)$ for LO (HO) case [8,9]. For the IO case, the values of the solar oscillation parameters $\left(\theta_{12}\right.$ and $\left.\Delta m_{21}^{2}\right)$ remain the same and for the remaining oscillation parameters, we consider $\theta_{13}=8.53^{\circ}, \Delta m_{31}^{2}=-2.42 \times 10^{-3} \mathrm{eV}^{2}$, and $\theta_{23}=42.1^{\circ}$ $\left(47.9^{\circ}\right)$ for $\mathrm{LO}(\mathrm{HO})$ scenario $[8,9]$. For the SI case, there is a clear separation between the black (LO) and red (HO) ellipses for both NO and IO. Once we introduce the LIV parameters $a_{e \mu}$ (left panel) and $a_{e \tau}$ (middle panel) one at-a-time, the LO and HO blobs show significant overlap among each other for both possible mass orderings. It suggest that in presence of a single LIV parameter, the $\theta_{23}$ octant separation capability of DUNE may get deteriorated significantly, which we confirm with the help of octant sensitivity plots in the next section. Interestingly, when both the LIV parameters $a_{e \mu}$ and $a_{e \tau}$ are present together in the scenario (see right panel of Fig. 2), the amount of overlap between the LO and HO blobs gets reduced considerably as compared the single LIV parameter case. This feature is consistent with our previous discussion in Sect. 2 (in connection with Eq. 25) that when both the LIV parameters $a_{e \mu}$ and $a_{e \tau}$ are present together, they may cancel out the impact of each other to a significant extent. In fact, we corroborate this reality while showing DUNE's octant discovery potential in our results section.

\section{Our results}

In this section, we present our sensitivity results. We start the discussion by showing the octant discovery potential of DUNE as a function of true values of the standard CP phase $\delta$ for both SI and SI+LIV schemes.

\subsection{Octant discovery potential as a function of true $\delta$}

Figure 3 exhibits the sensitivity (in terms of $\Delta \chi^{2}$ ) for excluding the wrong octant as a function of the true values of the standard Dirac CP phase $\delta$. The top (bottom) panels show the results assuming NO-LO (NO-HO) as the true choice with $\theta_{23}^{\text {true }}=42.3^{\circ}\left(47.7^{\circ}\right)$ for LO (HO) case. The left (mid- 


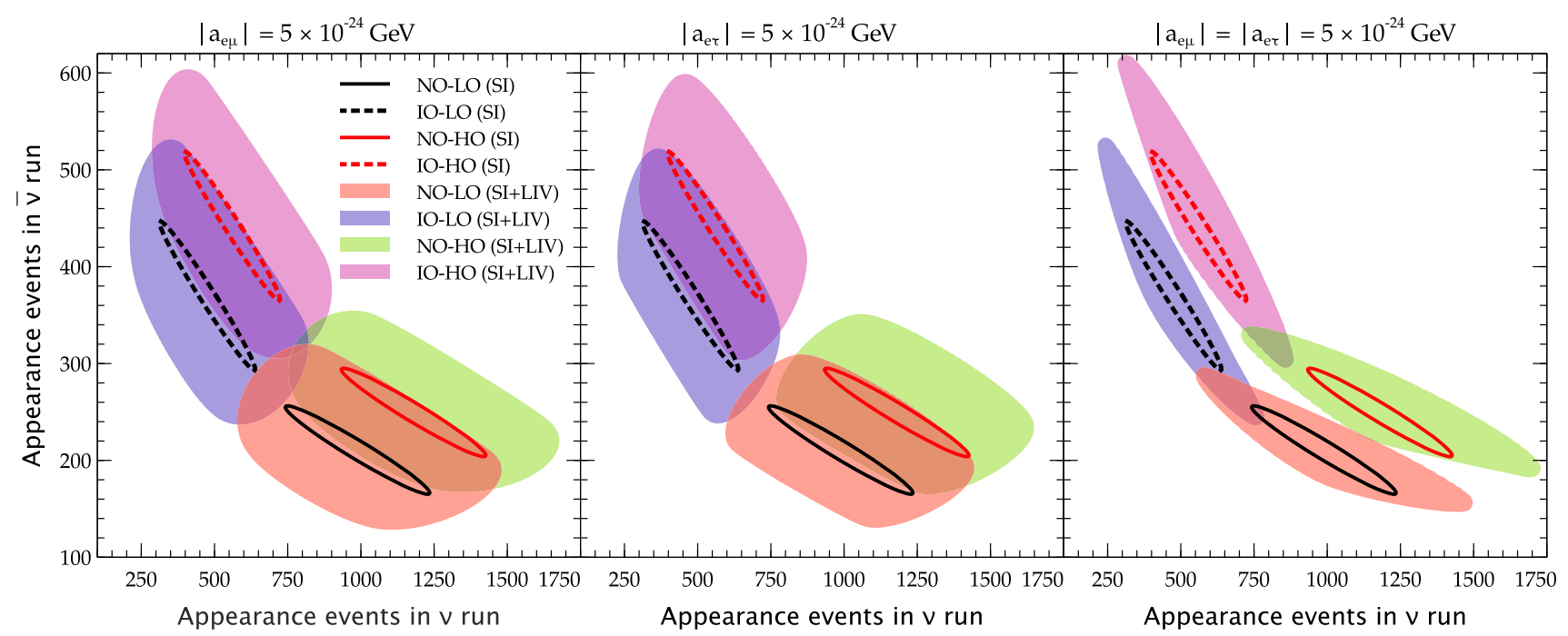

Fig. 2 Bi-event plots for DUNE. The standard interaction (SI) case is represented by the solid/dashed ellipses, which are obtained varying the standard CP phase $\delta$ in the range $[-\pi, \pi]$. The colored blobs denote the presence of LIV on top of the SI. These blobs are generated varying the CP phases: $\delta, \varphi_{e \mu}$ (left panel), $\delta, \varphi_{e \tau}$ (middle panel), $\delta, \varphi_{e \mu}$, and $\varphi_{e \tau}$ (right panel). In all the cases, $\mathrm{CP}$ phases are allowed to vary in their entire ranges of $[-\pi, \pi]$. In all the panels, the strength of the LIV parameter is taken to be $5 \times 10^{-24} \mathrm{GeV}$. For both SI and SI+LIV, we consider four possible cases: two possible mass orderings (NO and IO) together with two possible octants ( $\mathrm{LO}$ and $\mathrm{HO}$ ), as shown in the legends dle) panels consider the individual LIV parameter $a_{e \mu}\left(a_{e \tau}\right)$, while the right panels depict the case when both the LIV parameters are present together in the analysis. The black line in each panel represents the octant sensitivity for the SI case, while the four colored lines show the octant sensitivity for the SI+LIV framework considering four different true values of $\varphi_{e \mu}$ (left panel), $\varphi_{e \tau}$ (middle panel), and $\varphi_{e \mu}, \varphi_{e \tau}$ (right panel), as mentioned in the legends. In all the panels, the strength of the true LIV parameter is taken to be $5 \times 10^{-24} \mathrm{GeV}$. As discussed in Sect. 3, in the test dataset, $\theta_{23}^{\text {test }}$ has been marginalized over its all possible values in the wrong/opposite octant including the maximal value $\left(45^{\circ}\right)$. In the SI case, we perform the marginalization over $\delta^{\text {test }}$ in its entire range of $[-\pi, \pi]$, while in the SI+LIV scheme, we marginalize over both $\delta^{\text {test }}$ and $\varphi_{e \beta}^{\text {test }}$ (where $\beta$ can be $\mu$ or $\tau$ ) in their full parameter space of $[-\pi, \pi]$. We also marginalize over $\left|a_{e \beta}\right|^{\text {test }}$ in the range of $5 \times 10^{-25} \mathrm{GeV}$ to $5 \times 10^{-23}$ $\mathrm{GeV}$ as mentioned in Sect. 3. We note the following features from Fig. 3.

- For true LO (see top panels), the octant sensitivity for the SI case lies roughly between $4.5 \sigma$ to $5.2 \sigma$ depending on the true value of $\delta$. In presence of $\left|a_{e \mu}\right|$ (true) $=5 \times 10^{-24}$ $\mathrm{GeV}$, the sensitivity can be as low as $\sim 1 \sigma$ depending on the value of true $\delta$ and true $\varphi_{e \mu}$ (see top left panel). Similar degradation in the sensitivity is also observed in presence of $a_{e \tau}$ (see top middle panel). Since the standard CP phase $\delta$, as well as the LIV CP phases $\varphi_{e \mu}$ and $\varphi_{e \tau}$ are still undetermined, such spoiling of octant sensitivities is very much possible.

- For true HO (see bottom panels), the octant sensitivity for the SI case is relatively lower (approximately $2.2 \sigma$ to $2.7 \sigma)$. Here, in presence of $a_{e \mu}$ or $a_{e \tau}$, one can observe similar reduction in the sensitivity as we notice in the true LO case. The sensitivity may decrease to very small values $(\lesssim 1 \sigma)$ for many choices of true values of $\delta$ and $\varphi_{e \mu / e \tau}$ (see bottom left and bottom middle panels).

- In the right panels, in presence of both the LIV parameters $a_{e \mu}$ and $a_{e \tau}$ with the same magnitude, the worsening in the octant sensitivity is significantly less than what we observe for the single LIV parameter case. For true LO $(\mathrm{HO})$, the sensitivity does not go below $\sim 3.2 \sigma(1.5 \sigma)$. As we have discussed in Sects. 2 and 4, this is due to the fact that $a_{e \mu}$ and $a_{e \tau}$ effectively nullify the impact of each other to a significant extent (due to the presence of a relative sign between the $\Delta P_{\mu e}\left(a_{e \mu}\right)$ and $\Delta P_{\mu e}\left(a_{e \tau}\right)$ terms in Eq. 25). This very interesting and counterintuitive impact of LIV on octant sensitivity is discussed for the first time in the present work.

- The $\Delta \chi^{2}$ curves in presence of LIV have prominent peaks, which are more apparent for true LO. In presence of the LIV parameter $a_{e \mu}$, the choice of CP-conserving true values of $\varphi_{e \mu}=0, \pi$ (i.e., red and green curves, respectively) roughly produces peaks around maximal CP-violating true values of $\delta \simeq \pi / 2,-\pi / 2$, respectively. Conversely, maximal CP-violating true values of $\varphi_{e \mu}=\pi / 2,-\pi / 2$ (i.e., blue and magenta curves, respec- 


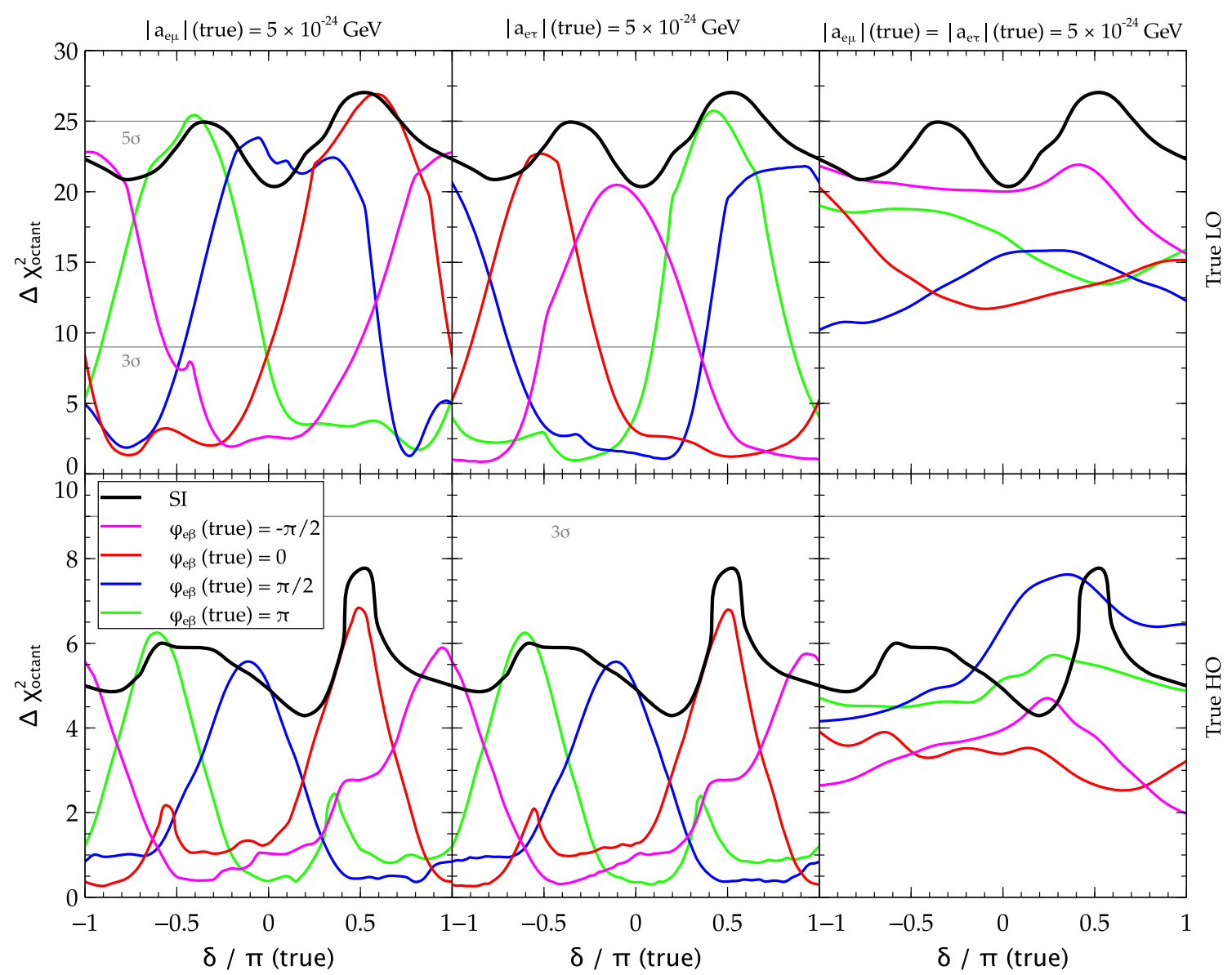

Fig. 3 Discovery potential of the true octant as a function of true $\delta$. In top (bottom) panels, we assume NO-LO (NO-HO) as the true choice with $\theta_{23}^{\text {true }}=42.3^{\circ}\left(47.7^{\circ}\right)$ as benchmark value for $\mathrm{LO}(\mathrm{HO})$ case. The left (middle) panels are for the individual LIV parameter $a_{e \mu}\left(a_{e \tau}\right)$, while the right panels deal with the case when both the LIV parameters are present simultaneously. In each panel, the black curve shows the result for the SI case, while the four colored lines depict the sen- sitivity for the SI+LIV scheme considering four different true values of $\varphi_{e \mu}$ (left panel), $\varphi_{e \tau}$ (middle panel), and $\varphi_{e \mu}, \varphi_{e \tau}$ (right panel), as mentioned in the legends. In all the panels, the strength of the true LIV parameter is assumed to be $5 \times 10^{-24} \mathrm{GeV}$. See text for marginalization and other details. Note that the $y$-axis ranges are different in top and bottom panels tively) approximately produce peaks near CP-conserving true values of $\delta \simeq 0, \pm \pi$, respectively. This trend can also be observed in presence of $a_{e \tau}$ with the location of the peaks interchanged. In presence of both $a_{e \mu}$ and $a_{e \tau}$, such a prominent feature is not noticed.

\subsection{Octant discovery potential in $\left[\sin ^{2} \theta_{23}-\delta\right]$ (true) plane}

The exact value of $\sin ^{2} \theta_{23}$ is still to be determined. Therefore, we consider all the allowed values of $\sin ^{2} \theta_{23}$ and portray in Fig. 4, the discovery potential of the true octant in the parameter space of true $\sin ^{2} \theta_{23}-$ true $\delta$ at $2 \sigma$ (solid blue curves), $3 \sigma$ (dashed magenta curves), and $4 \sigma$ (dotted black curves) confidence levels ( 1 d.o.f.) such that $\sigma=\sqrt{\Delta \chi^{2}}$. We explore four different schemes: the SI case (top left panel), the SI+LIV case with $a_{e \mu}$ (top right panel), the SI+LIV scheme with $a_{e \tau}$ (bottom right panel), and the SI+LIV framework with both $a_{e \mu}$ and $a_{e \tau}$ present together in the simulation (bottom left panel). In all the panels, the strength of the true LIV parameter is assumed to be $5 \times 10^{-24} \mathrm{GeV}$. Here, we assume NO both in data and theory. In case of SI, we calculate the $\Delta \chi^{2}$ by marginalizing over test $\delta(\in[-\pi, \pi])$ and test $\theta_{23}$ over the wrong octant ${ }^{7}$. In the SI+LIV schemes, we additionally marginalize over $\left|a_{e \mu}\right|^{\text {test }}$, the true and test values of the new dynamical CP phase $\varphi_{e \mu}$ (top left panel) and $\left|a_{e \tau}\right|^{\text {test }}, \varphi_{e \tau}^{\text {true }}$, $\varphi_{e \tau}^{\text {test }}$ (bottom right panel). In bottom left panel, we marginalize over $\left|a_{e \mu}\right|^{\text {test }},\left|a_{e \tau}\right|^{\text {test }}$, and the true and test values of the additional CP phases $\varphi_{e \mu}$ and $\varphi_{e \tau}$ in their entire allowed range of $[-\pi, \pi]$. It is apparent that in presence of the indi-

\footnotetext{
${ }^{7}$ For instance, for every true $\theta_{23}$ in the LO (i.e., $\theta_{23}^{\text {true }}<45^{\circ}$ ), the test $\theta_{23}$ is marginalized over the entire allowed range in $\mathrm{HO}$ (i.e., $\theta_{23}^{\text {test }} \in$ $\left.\left[45^{\circ}, 50.7^{\circ}\right]\right)$. Similarly, for every true $\theta_{23}$ in the $\mathrm{HO}\left(\right.$ i.e., $\theta_{23}^{\text {true }}>45^{\circ}$ ), the test $\theta_{23}$ is marginalized over the entire allowed range in $\mathrm{LO}$ (i.e., $\left.\theta_{23}^{\text {test }} \in\left[41.8^{\circ}, 45^{\circ}\right]\right)$.
} 
Fig. 4 Octant discovery potential in $\left[\sin ^{2} \theta_{23}-\delta\right]$ (true) plane at $2 \sigma$ (solid blue curves), $3 \sigma$ (dashed magenta curves), and $4 \sigma$ (dotted black curves) confidence levels (1 d.o.f.) assuming $\mathrm{NO}$ both in data and theory. We consider four different scenarios: the SI case (top left panel), the SI+LIV case with $a_{e \mu}$ (top right panel), the SI+LIV scheme with $a_{e \tau}$ (bottom right panel), and the SI+LIV framework with both $a_{e \mu}$ and $a_{e \tau}$ present together (bottom left panel). In all the panels, the strength of the true LIV parameter is assumed to be $5 \times 10^{-24} \mathrm{GeV}$. See text for marginalization and other details
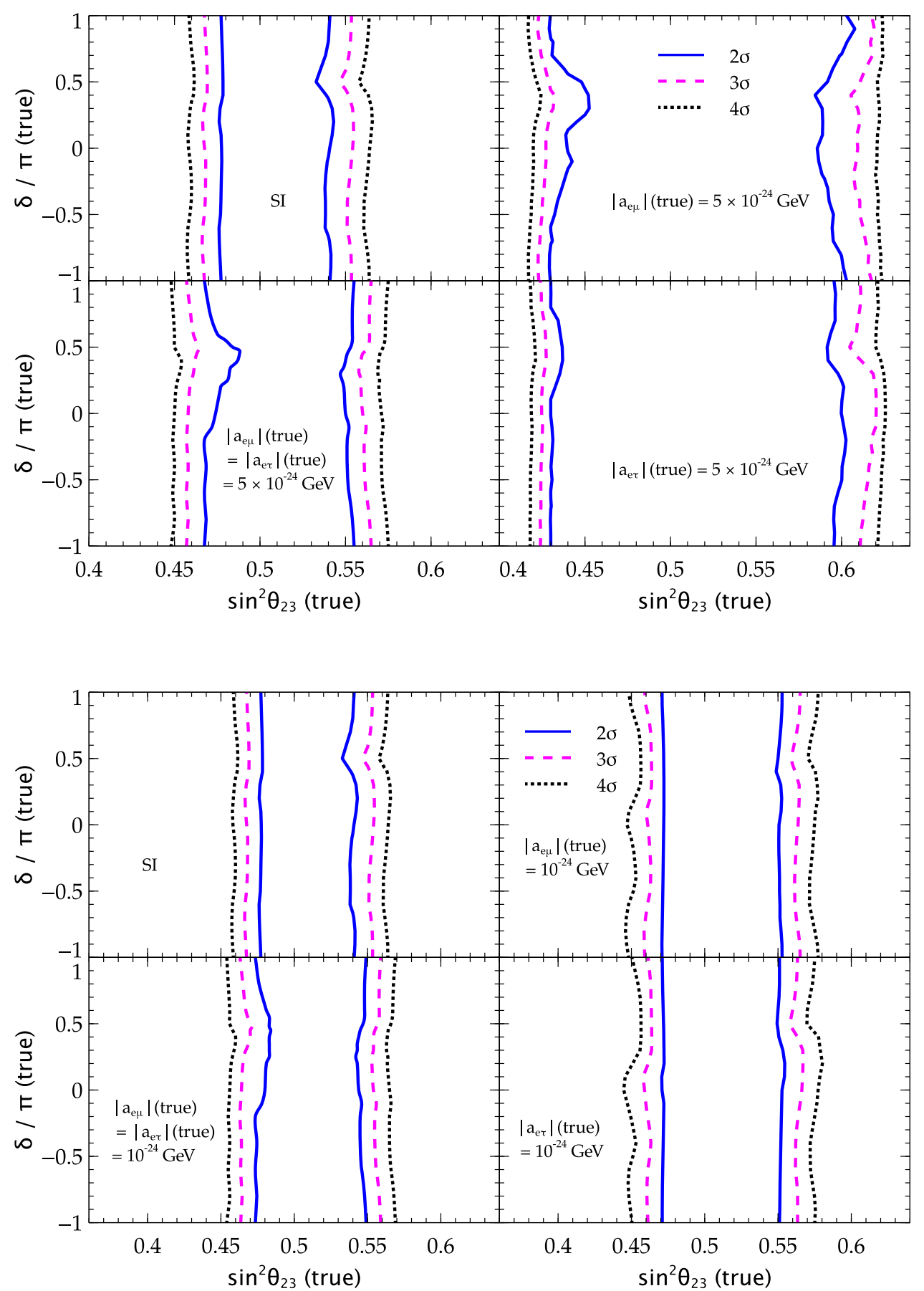

Fig. 5 Octant discovery potential in $\left[\sin ^{2} \theta_{23}-\delta\right]$ (true) plane at $2 \sigma$ (solid blue curves), $3 \sigma$ (dashed magenta curves), and $4 \sigma$ (dotted black curves) confidence levels (1 d.o.f.) assuming $\mathrm{NO}$ both in data and theory. We consider four different scenarios: the SI case (top left panel), the SI+LIV case with $a_{e \mu}$ (top right panel), the SI+LIV scheme with $a_{e \tau}$ (bottom right panel), and the SI+LIV framework with both $a_{e \mu}$ and $a_{e \tau}$ present together (bottom left panel). In all the panels, the strength of the true LIV parameter is assumed to be $10^{-24} \mathrm{GeV}$. See text for marginalization and other details vidual LIV parameter $a_{e \mu}$ (top right panel) or $a_{e \tau}$ (bottom right panel), the sensitivity towards the octant of $\theta_{23}$ gets reduced considerably as compared to the SI case (top left panel). In such cases, the octant of $\theta_{23}$ can only be resolved at $3 \sigma$ confidence level if the true value of $\theta_{23}$ turns out to be at least $5-7^{\circ}$ away from maximal mixing $\left(45^{\circ}\right)$ for any choices of $\delta$ and $\varphi_{e \mu / e \tau}$. When both the LIV parameters $a_{e \mu}$ and $a_{e \tau}$ are present together in the simulation (see bottom left panel), they cancel their effect to a large extent, and we see a very slight deterioration in the octant sensitivity as compared to the SI case. We observe similar feature in the previous section as well.

In Fig. 5 (Fig. 6), we portray the same assuming the strength of the true LIV parameter to be $10^{-24} \mathrm{GeV}\left(10^{-23}\right.$ $\mathrm{GeV}$ ). It is evident from Eq. 13 that the impact of the LIV in $v_{\mu} \rightarrow v_{e}$ transition channel is proportional to the strength of 
Fig. 6 Octant discovery potential in [ $\sin ^{2} \theta_{23}-\delta$ ] (true) plane at $2 \sigma$ (solid blue curves), $3 \sigma$ (dashed magenta curves), and $4 \sigma$ (dotted black curves) confidence levels (1 d.o.f.) assuming NO both in data and theory. We consider four different scenarios: the SI case (top left panel), the SI+LIV case with $a_{e \mu}$ (top right panel), the SI+LIV scheme with $a_{e \tau}$ (bottom right panel), and the SI+LIV framework with both $a_{e \mu}$ and $a_{e \tau}$ present together (bottom left panel). In all the panels, the strength of the true LIV parameter is assumed to be $10^{-23} \mathrm{GeV}$. See text for marginalization and other details

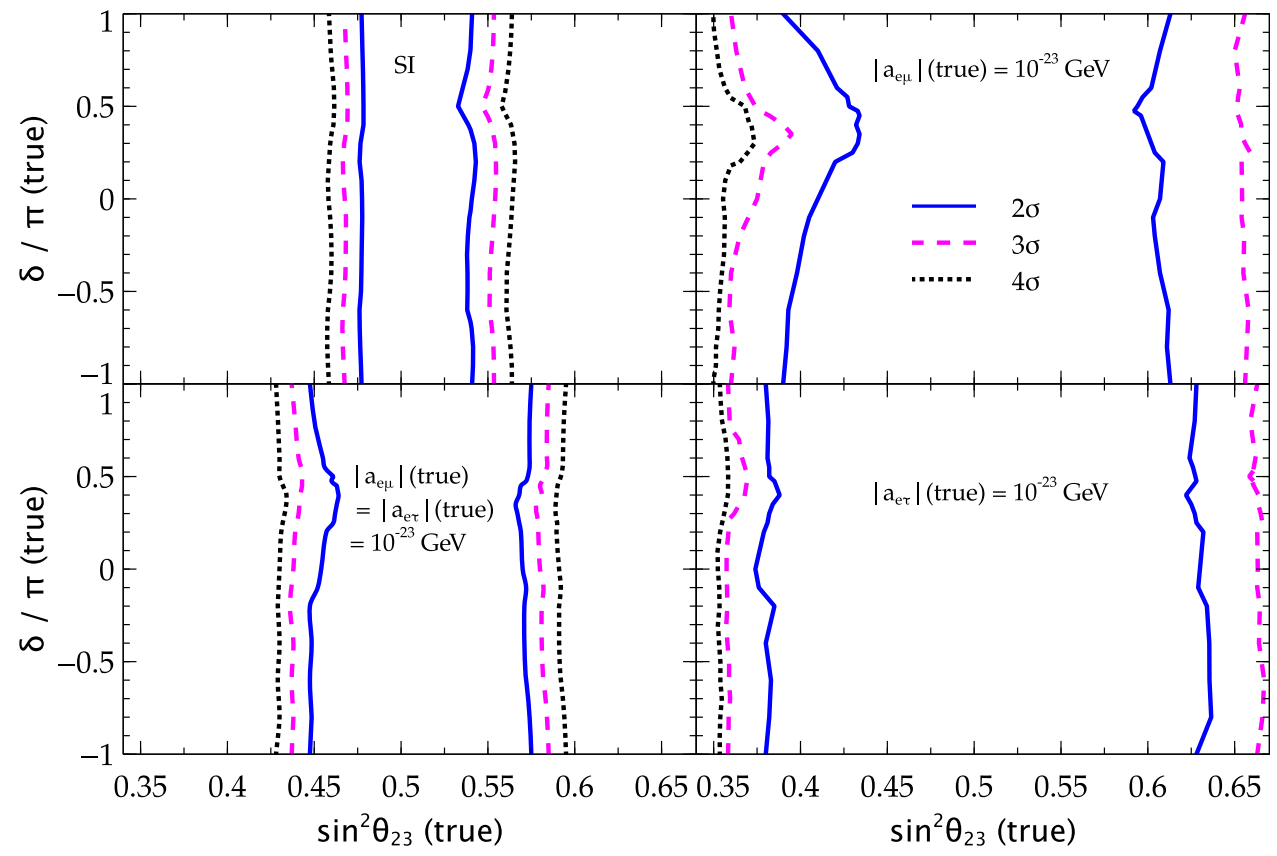

the LIV parameter $\left|a_{e \beta}\right|$ (where $\beta$ can be $\mu$ or $\tau$ ). For this reason, as we decrease the strength of the LIV parameter in data from $5 \times 10^{-24} \mathrm{GeV}$ (see Fig. 4) to $10^{-24} \mathrm{GeV}$ (see Fig. 5), we see a very minimal deterioration in the octant discovery potential in the SI+LIV case as compared to the SI case. On the contrary, if we increase the strength of the true LIV parameter from $5 \times 10^{-24} \mathrm{GeV}$ (see Fig. 4) to $10^{-23} \mathrm{GeV}$ (see Fig. 6), we observe a huge deterioration in the octant discovery potential in the SI+LIV scheme as compared to the SI scheme. In Fig. 6, when we consider one LIV parameter at-a-time (top and bottom right panels), the worsening in the octant discovery potential is so large that even at $2 \sigma$ confidence level, the true values of $\sin ^{2} \theta_{23}$ for which DUNE would be able to resolve the octant, almost go beyond the present $3 \sigma$ allowed range.

\subsection{Octant discovery potential as a function of LIV strength}

So far, we have shown our results for few benchmark true values of the LIV parameters $\left|a_{e \mu}\right|$ and $\left|a_{e \tau}\right|$. Now, it would be quite interesting to see how the octant discovery potential gets modified if we vary the strength of the LIV parameters $\left|a_{e \mu}\right|$ (true) and $\left|a_{e \tau}\right|$ (true). We present the result of this analysis in Fig. 7, which exhibits the discovery potential of the $\theta_{23}$ octant as a function of the strength of the true LIV parameter $\left|a_{e \mu}\right|\left(\left|a_{e \tau}\right|\right)$ in left (right) panel assuming NO both in data and theory. In both the panels, we marginalize over the test values of $\theta_{23}$ in the wrong octant and the standard CP phase $\delta$ (both true and test) in its full range of $[-\pi, \pi]$. We marginalize over test choices of $\left|a_{e \mu}\right|\left(\left|a_{e \tau}\right|\right)$ in the left (right) panel. In addition to this, in the left (right) panel, the true and test values of the new CP phase $\varphi_{e \mu}\left(\varphi_{e \tau}\right)$ have been marginalized away in the entire range of $[-\pi, \pi]$. We show the results at three different confidence levels ( 1 d.o.f.): $2 \sigma$ (solid blue curves), $3 \sigma$ (dashed magenta curves), and $4 \sigma$ (dotted black curves). It is clear that as the strength of the LIV parameter increases, the discovery potential of the true octant gets deteriorated gradually. We notice that as the strength of the LIV parameters approaches towards $10^{-25} \mathrm{GeV}$, the sensitivities slowly get improved and attain the values which we obtain in the SI case.

\subsection{Reconstruction of the $\mathrm{CP}$ phases}

In the previous subsections, we discuss in detail how the standard CP phase $\delta$ and the new dynamical CP phase $\varphi_{e \mu / e \tau}$ would affect the measurement of the octant of $\theta_{23}$ in DUNE. In this subsection, we explore the capability of DUNE in reconstructing the true values of the $\mathrm{CP}$ phases $\delta$ and $\varphi_{e \beta}$ (where $\beta$ can be $\mu$ or $\tau$ ). Figure 8 shows the reconstructed regions for the two CP phases $\delta$ and $\varphi_{e \mu}$ (top panels) at $1 \sigma$, $2 \sigma$, and $3 \sigma$ ( 1 d.o.f.) confidence levels assuming NO both in data and theory. The bottom panels in Fig. 8 portray the same for the two CP phases $\delta$ and $\varphi_{e \tau}$. The two left panels refer to the typical CP-conserving true values of the phases $[0,0]$, while the two right panels deal with the illustrative $\mathrm{CP}$-violating true values of the phases $[-\pi / 2,-\pi / 2]$. In all the panels, the strength of the true LIV parameter is assumed to be $5 \times 10^{-24} \mathrm{GeV}$, and we marginalize over test choices of LIV parameter in the fit. While generating the prospec- 


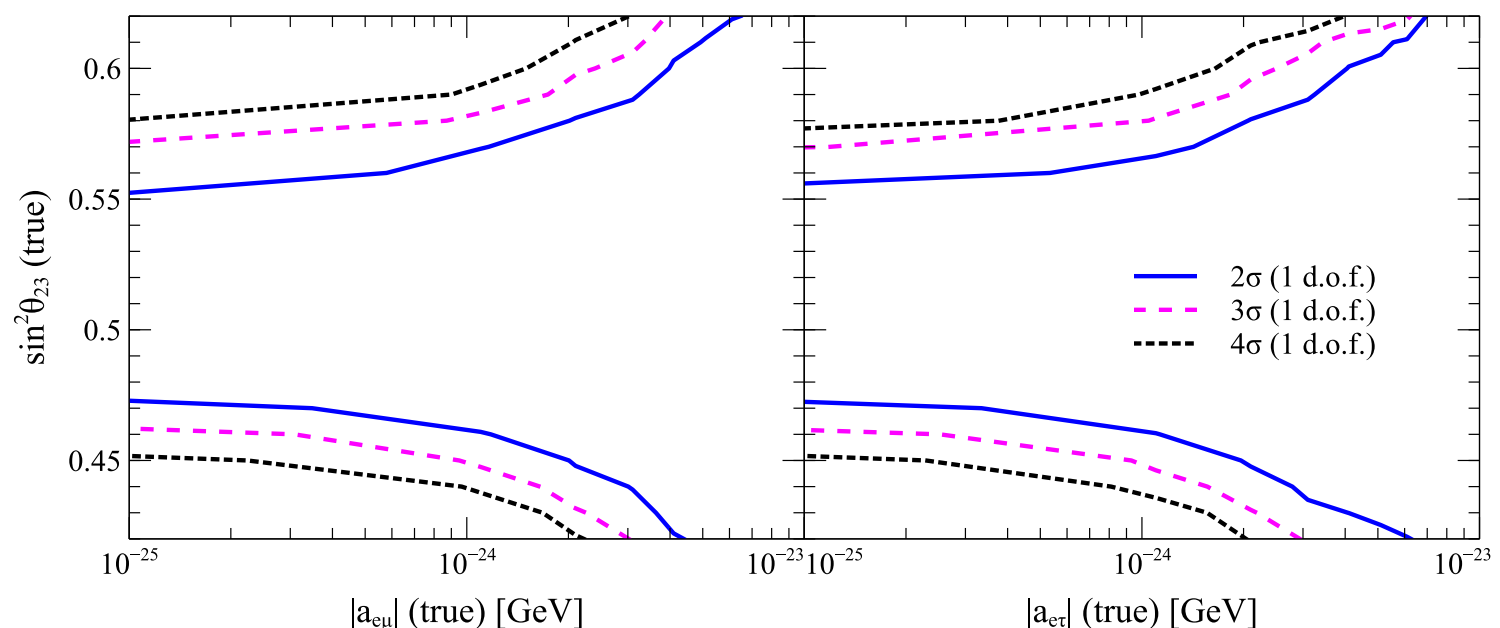

Fig. 7 Deterioration of the $\theta_{23}$ octant discovery potential as a function of the strength of the true LIV parameter $\left|a_{e \mu}\right|\left(\left|a_{e \tau}\right|\right)$ in left (right) panel assuming NO both in data and theory. In both the panels, we marginalize over the test values of $\theta_{23}$ in the wrong octant and the standard CP phase $\delta$ (both true and test) in its full range of $[-\pi, \pi]$. We marginalize over test choices of $\left|a_{e \mu}\right|\left(\left|a_{e \tau}\right|\right)$ in the left (right) panel. In addition to this, in the left (right) panel, the true and test values of the new CP phase $\varphi_{e \mu}\left(\varphi_{e \tau}\right)$ have been marginalized away. We show the results at three different confidence levels (1 d.o.f.): $2 \sigma$ (solid blue curves), $3 \sigma$ (dashed magenta curves), and $4 \sigma$ (dotted black curves)

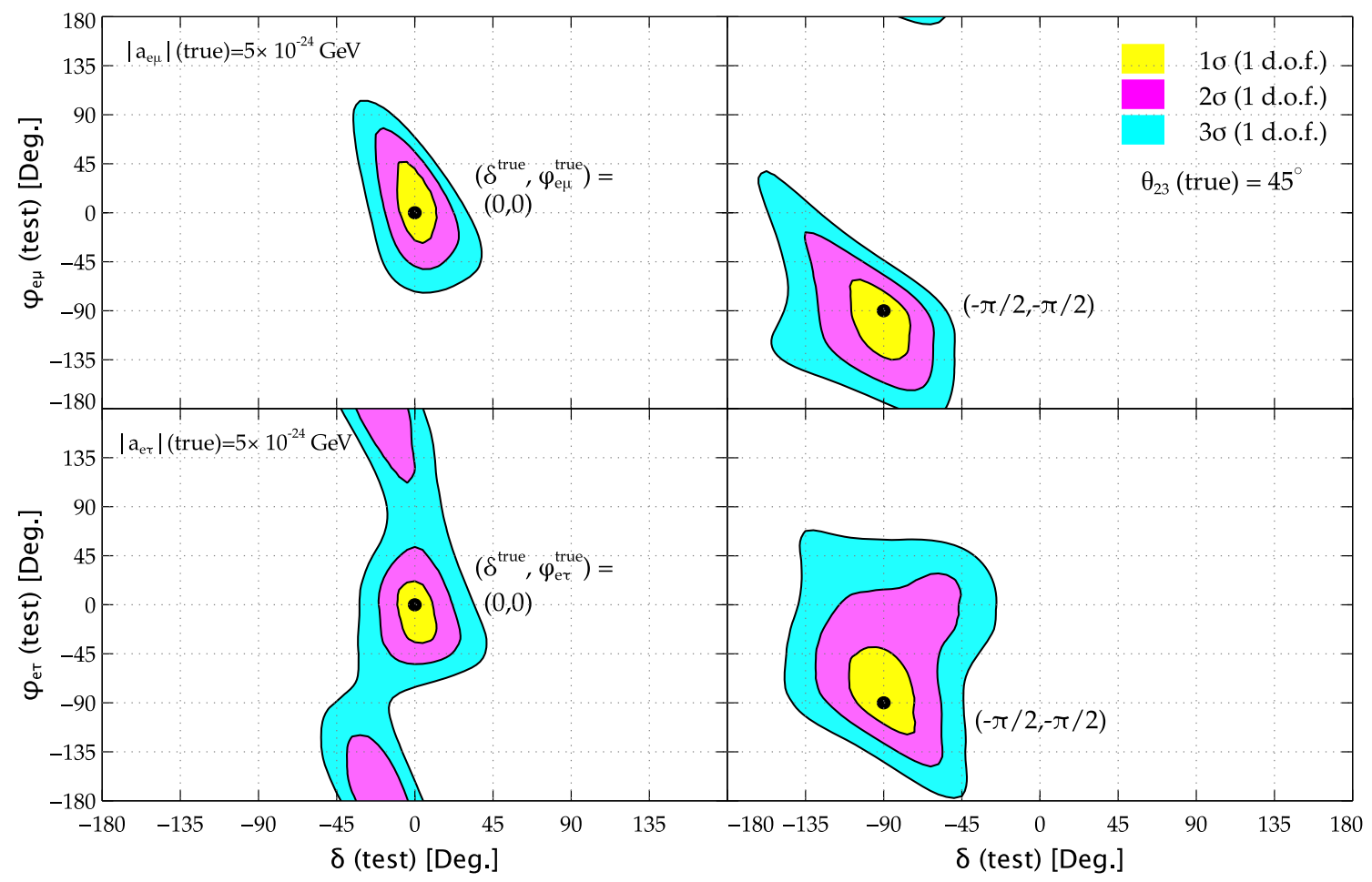

Fig. 8 Reconstructed regions for the two CP phases $\delta$ and $\varphi_{e \mu}$ (top panels) at $1 \sigma, 2 \sigma$, and $3 \sigma$ ( 1 d.o.f.) confidence levels assuming NO both in data and theory. The bottom panels show the same for the two CP phases $\delta$ and $\varphi_{e \tau}$. The two left (right) panels refer to the representative true values of the phases $[0,0]([-\pi / 2,-\pi / 2])$. In all the panels, the strength of the true LIV parameter is assumed to be $5 \times 10^{-24} \mathrm{GeV}$, and we marginalize over test choices of LIV parameter in the fit. We consider $\theta_{23}^{\text {true }}=45^{\circ}$ and marginalize over $\theta_{23}^{\text {test }}$ in the range $\left[41.8^{\circ}, 50.7^{\circ}\right]$ in the fit 
Table 1 The typical $1 \sigma$ allowed ranges around the true values of the $\mathrm{CP}$ phases $\delta$ and $\varphi_{e \beta}$ (where $\beta$ can be $\mu$ or $\tau$ ). Here, we assume the strength of the true LIV parameters $\left|a_{e \mu}\right|$ and $\left|a_{e \tau}\right|$ to be $5 \times 10^{-24} \mathrm{GeV}$, and we marginalize over the test choices of the corresponding LIV parameters in the fit

\begin{tabular}{llr}
\hline True values & $1 \sigma$ range in $\delta^{\text {test }}($ Deg. $)$ & $1 \sigma$ range in $\varphi^{\text {test }}($ Deg. $)$ \\
\hline$\left(\delta, \varphi_{e \mu}\right)=(0,0)$ & $-8^{\circ} \lesssim \delta^{\text {test }} \lesssim 12^{\circ}$ & $-25^{\circ} \lesssim \varphi_{e \mu}^{\text {test }} \lesssim 38^{\circ}$ \\
$\left(\delta, \varphi_{e \tau}\right)=(0,0)$ & $-9^{\circ} \lesssim \delta^{\text {test } \lesssim 10^{\circ}}$ & $-33^{\circ} \lesssim \varphi_{e \tau}^{\text {test }} \lesssim 21^{\circ}$ \\
$\left(\delta, \varphi_{e \mu}\right)=(-\pi / 2,-\pi / 2)$ & $-108^{\circ} \lesssim \delta^{\text {test }} \lesssim-78^{\circ}$ & $-131^{\circ} \lesssim \varphi_{e \mu}^{\text {test }} \lesssim-74^{\circ}$ \\
$\left(\delta, \varphi_{e \tau}\right)=(-\pi / 2,-\pi / 2)$ & $-105^{\circ} \lesssim \delta^{\text {test } \lesssim-73^{\circ}}$ & $-108^{\circ} \lesssim \varphi_{e \tau}^{\text {test }} \lesssim-42^{\circ}$ \\
\hline
\end{tabular}

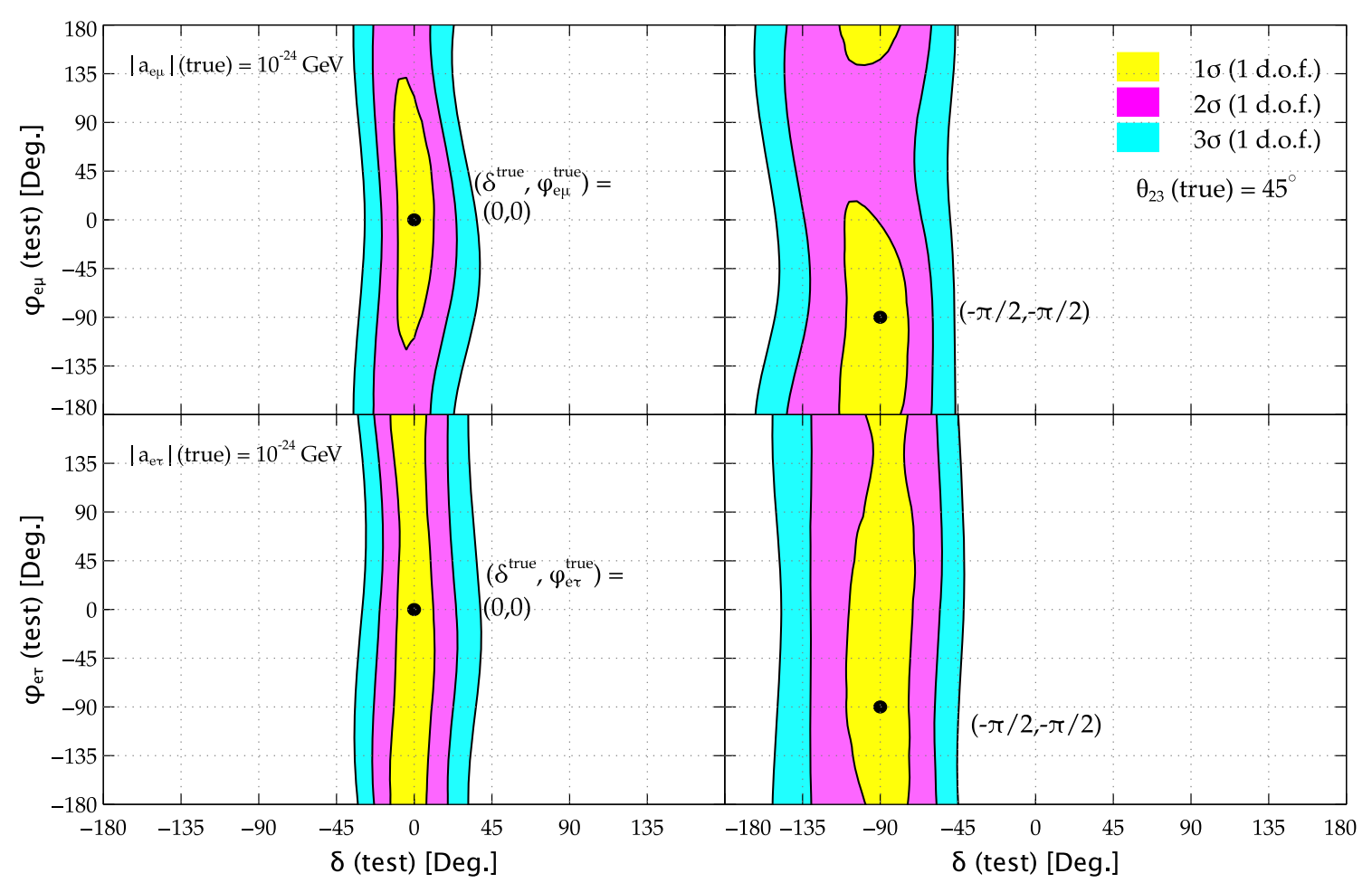

Fig. 9 Reconstructed regions for the two CP phases $\delta$ and $\varphi_{e \mu}$ (top panels) at $1 \sigma, 2 \sigma$, and $3 \sigma$ (1 d.o.f.) confidence levels assuming $\mathrm{NO}$ both in data and theory. The bottom panels show the same for the two CP phases $\delta$ and $\varphi_{e \tau}$. The two left (right) panels refer to the representative true values of the phases $[0,0]([-\pi / 2,-\pi / 2])$. In all the panels, the strength of the true LIV parameter is assumed to be $10^{-24} \mathrm{GeV}$, and we marginalize over test choices of LIV parameter in the fit. We consider $\theta_{23}^{\text {true }}=45^{\circ}$ and marginalize over $\theta_{23}^{\text {test }}$ in the range $\left[41.8^{\circ}, 50.7^{\circ}\right]$ in the fit tive data for DUNE, we consider the true value of $\theta_{23}$ to be $45^{\circ}$ and in the fit, we marginalize over the test values of $\theta_{23}$ in its $3 \sigma$ allowed range of $41.8^{\circ}$ to $50.7^{\circ}$. DUNE can measure the $\mathrm{CP}$ phases $\delta$ and $\varphi_{e \mu}$ quite efficiently providing a unique reconstructed region around $\delta^{\text {true }}=\varphi_{e \mu}^{\text {true }}=0^{\circ}$ at $3 \sigma$ confidence level (see top left panel). But in presence of $a_{e \tau}$ (see bottom left panel), the reconstruction becomes quite poor for $\varphi_{e \tau}$ at $2 \sigma$ and above. For maximal CP-violating choices $(-\pi / 2,-\pi / 2)$ of the true CP phases (see top right and bottom right panels), the reconstruction of $\delta^{\text {true }}$ becomes slightly worse as compared to the CP-conserving scenarios at $1 \sigma$ confidence level, while the reconstruction of $\varphi_{e \mu}^{\text {true }}$ and $\varphi_{e \tau}^{\text {true }}$ remains more or less same. In Table 1 , we mention the typical $1 \sigma$ allowed ranges around the true values of the $\mathrm{CP}$ phases $\delta$ and $\varphi_{e \beta}$ (where $\beta$ can be $\mu$ or $\tau$ ). Here, we assume the strength of the true LIV parameters $\left|a_{e \mu}\right|$ and $\left|a_{e \tau}\right|$ to be $5 \times 10^{-24} \mathrm{GeV}$, and we marginalize over the test choices of the corresponding LIV parameters in the fit. We have also checked that the reconstruction of the $\mathrm{CP}$ phases becomes worse as $\theta_{23}$ deviates from the maximal mixing.

In Figs. 9 and 10, we show the performance of DUNE in reconstructing the true values of the $\mathrm{CP}$ phases $\delta$ and $\varphi_{e} \beta$ (where $\beta$ can be $\mu$ or $\tau$ ) considering the strength of the true LIV parameter to be $10^{-24} \mathrm{GeV}$ and $10^{-23} \mathrm{GeV}$, respec- 


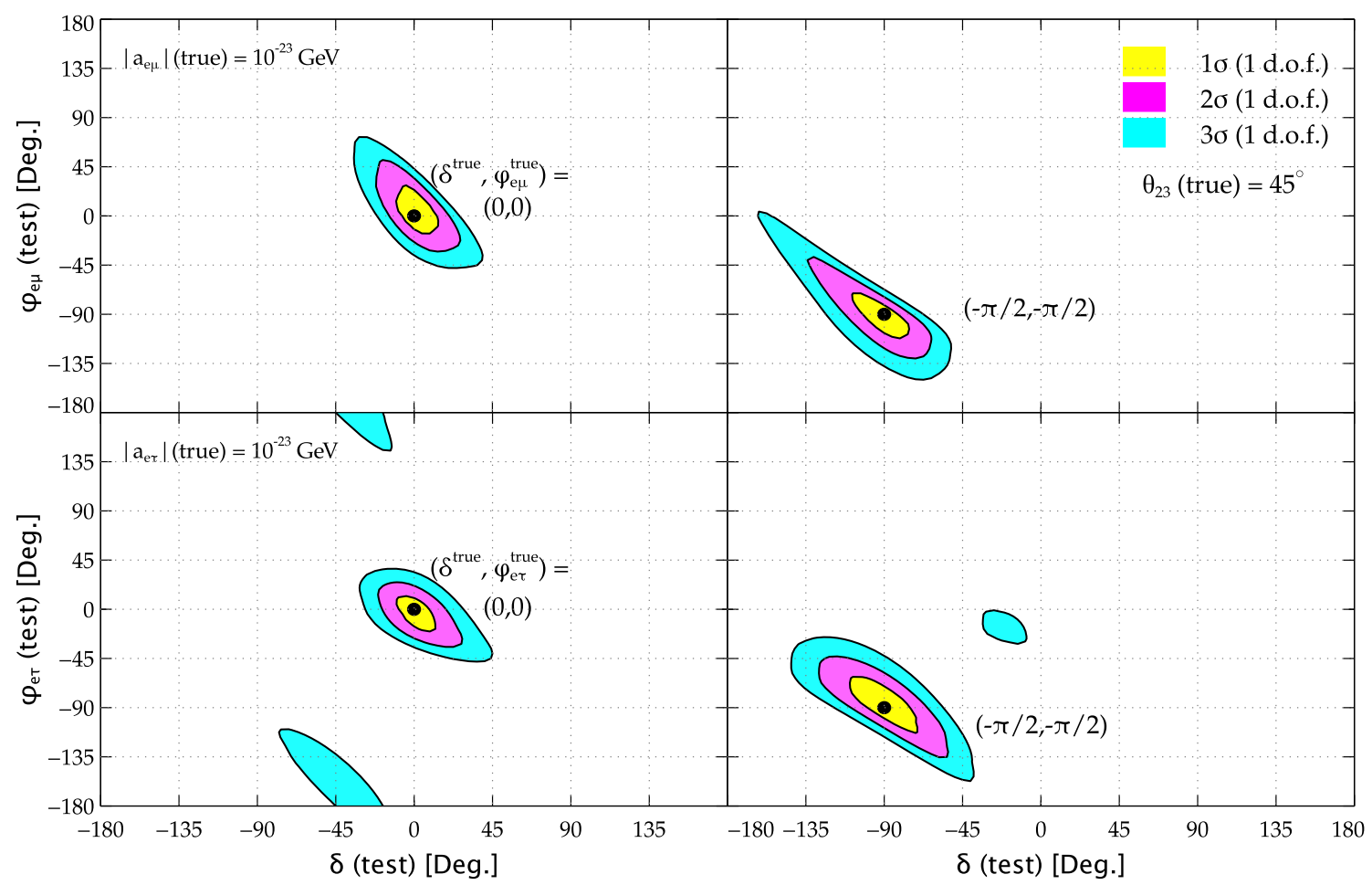

Fig. 10 Reconstructed regions for the two CP phases $\delta$ and $\varphi_{e \mu}$ (top panels) at $1 \sigma, 2 \sigma$, and $3 \sigma$ (1 d.o.f.) confidence levels assuming NO both in data and theory. The bottom panels show the same for the two CP phases $\delta$ and $\varphi_{e \tau}$. The two left (right) panels refer to the representative true values of the phases $[0,0]([-\pi / 2,-\pi / 2])$. In all the panels, the

tively. As expected, once we decrease the strength of the true LIV parameter $\left|a_{e \mu}\right| /\left|a_{e \tau}\right|$ from $5 \times 10^{-24} \mathrm{GeV}$ (see Fig. 8) to $10^{-24} \mathrm{GeV}$ (see Fig. 9), the reconstruction of the new dynamical CP phases $\varphi_{e \mu / e \tau}$ becomes quite impossible, and almost $75 \%$ values of $\varphi_{e \mu}$ and all the values of $\varphi_{e \tau}$ get allowed even at $1 \sigma$ confidence level. As far as the reconstruction of the standard CP phase $\delta$ is concerned, the allowed regions for $\delta$ remain almost the same in Figs. 8 and 9. When we increase the strength of the true LIV parameter $\left|a_{e \mu}\right| /\left|a_{e \tau}\right|$ in data from $5 \times 10^{-24} \mathrm{GeV}$ (see Fig. 8) to $10^{-23} \mathrm{GeV}$ (see Fig. 10), the reconstruction of the new dynamical CP phases $\varphi_{e \mu} / \varphi_{e \tau}$ gets improved significantly and the allowed regions for $\delta$ remain almost unaltered.

\section{Summary and conclusion}

We have a well-defined neutrino roadmap to resolve the remaining fundamental unknowns, in particular, the determination of neutrino mass ordering, the clear demonstration of leptonic CP-violation (CPV), and the precision measurement of the oscillation parameters with the help of upcoming high-precision long-baseline neutrino oscillation experiment strength of the true LIV parameter is assumed to be $10^{-23} \mathrm{GeV}$, and we marginalize over test choices of LIV parameter in the fit. We consider $\theta_{23}^{\text {true }}=45^{\circ}$ and marginalize over $\theta_{23}^{\text {test }}$ in the range $\left[41.8^{\circ}, 50.7^{\circ}\right]$ in the fit

DUNE. This experiment will perform a rigorous test of the three-flavor oscillation framework and play an important role to test the existence of various new physics scenarios if they at all exist in Nature. One such new physics scenario is Lorentz invariance violation (LIV).

In this paper, we study the impact of LIV in determining the octant of $\theta_{23}$ and in reconstructing the CP phases considering the DUNE as a case study. We discuss in detail how the two most relevant CPT-violating LIV parameters $a_{e \mu}\left(\equiv\left|a_{e \mu}\right| e^{i \varphi_{e \mu}}\right)$ and $a_{e \tau}\left(\equiv\left|a_{e \tau}\right| e^{i \varphi_{e \tau}}\right)$ affect neutrino and antineutrino appearance probabilities. These LIV parameters $\left(a_{e \mu}\right.$ or $\left.a_{e \tau}\right)$ introduce an additional interference term in $v_{\mu} \rightarrow v_{e}$ and $\bar{v}_{\mu} \rightarrow \bar{v}_{e}$ oscillation channels. This new interference term depends on both the standard CP phase $\delta$ and the new dynamical CP phase $\varphi_{e \mu / e \tau}$. This term gets summed up with the well-known interference term related to the standard $\mathrm{CP}$ phase $\delta$ and gives rise to new degeneracies among $\theta_{23}$, $\delta$, and $\varphi$. These new degeneracies spoil the measurement of octant of $\theta_{23}$. We show that for values of the LIV parameter (taken one at-a-time) as small as $\left|a_{e \mu}\right|=\left|a_{e \tau}\right|=5 \times 10^{-24}$ $\mathrm{GeV}$, the octant discovery potential of DUNE deteriorates considerably for unfavorable combinations of the two $\mathrm{CP}$ phases $\delta$ and $\varphi_{e \mu / e \tau}$. DUNE can only resolve the octant ambi- 
guity of $\theta_{23}$ at $3 \sigma$ confidence level for any choices of $\delta$ and $\varphi$ if $\theta_{23}$ turns out to be at least $5^{\circ}$ to $7^{\circ}$ away from maximal mixing. We also perform the analysis considering both the LIV parameters $a_{e \mu}$ and $a_{e \tau}$ together and observe for the first time that they largely nullify the effect of each other due to the apparent relative sign between the $a_{e \mu}$-term and the $a_{e \tau^{-}}$ term in the probability expressions. For this reason, DUNE can retrieve its octant resolution capability if both the LIV parameters $a_{e \mu}$ and $a_{e \tau}$ are present together in the analysis. We also study how the deterioration of the $\theta_{23}$ octant discovery potential varies with the magnitude of the LIV parameters in the $\left[\sin ^{2} \theta_{23}\right.$ (true) $-\left|a_{e \beta}\right|$ (true)] plane where $\beta$ can be $\mu$ or $\tau$. We also address how well DUNE can reconstruct the standard CP phase $\delta$ and the new dynamical CP phase $\varphi_{e \mu / e \tau}$. Our analysis reveals that the typical $1 \sigma$ uncertainty on $\delta$ is $10^{\circ}$ to $15^{\circ}$ and the same on $\varphi_{e \mu} / \varphi_{e \tau}$ is $25^{\circ}$ to $30^{\circ}$. So, at the end, we conclude that a small amount of Lorentz Invariance Violation (LIV) may affect the measurements of octant of 2-3 mixing angle and the CP phases at DUNE and we hope that our present study will be a valuable addition to the landscape of new physics scenarios beyond the standard three-neutrino oscillation paradigm which can be probed using the DUNE facility.

Acknowledgements S.K.A. is supported by the DST/INSPIRE Research Grant [IFA-PH-12] from the Department of Science and Technology (DST), India. S.K.A. and M.M. acknowledge the financial support from the Indian National Science Academy (INSA) Young Scientist Project [INSA/SP/YSP/144/2017/1578].

Data Availability Statement This manuscript has no associated data or the data will not be deposited. [Authors' comment: This is a sensitivity study of a future experiment and we have generated mock data to study the performance of the experiment. We have not used any external dataset in our study.]

Open Access This article is licensed under a Creative Commons Attribution 4.0 International License, which permits use, sharing, adaptation, distribution and reproduction in any medium or format, as long as you give appropriate credit to the original author(s) and the source, provide a link to the Creative Commons licence, and indicate if changes were made. The images or other third party material in this article are included in the article's Creative Commons licence, unless indicated otherwise in a credit line to the material. If material is not included in the article's Creative Commons licence and your intended use is not permitted by statutory regulation or exceeds the permitted use, you will need to obtain permission directly from the copyright holder. To view a copy of this licence, visit http://creativecomm ons.org/licenses/by/4.0/.

Funded by SCOAP ${ }^{3}$.
Appendix: Octant discovery potential with LIV in data only

Here, we explore the octant discovery potential of DUNE assuming the presence of LIV in data, but not in fit (theory). Fig. 11 shows the octant discovery potential of DUNE as a function of true $\delta$. In top (bottom) panels, we assume NO$\mathrm{LO}(\mathrm{NO}-\mathrm{HO})$ as the true choice with $\theta_{23}^{\text {true }}=42.3^{\circ}\left(47.7^{\circ}\right)$ as benchmark value for $\mathrm{LO}(\mathrm{HO})$ case. In all the panels, the data are generated assuming the strength of the true LIV parameter to be $5 \times 10^{-24} \mathrm{GeV}$, and we do not consider the LIV parameters in the fit (theory). The left (middle) panels are for the individual LIV parameter $a_{e \mu}\left(a_{e \tau}\right)$, while the right panels deal with the case when both the LIV parameters are present simultaneously. In each panel, the black curve shows the result for the SI case, while the four colored lines depict the sensitivity for the SI+LIV scheme considering four different true values of $\varphi_{e \mu}$ (left panel), $\varphi_{e \tau}$ (middle panel), and $\varphi_{e \mu}, \varphi_{e \tau}$ (right panel), as mentioned in the legends. We marginalize over $\theta_{23}^{\text {test }}$ in the wrong/opposite octant including the maximal value $\left(45^{\circ}\right)$ and $\delta^{\text {test }}$ in its entire range of $[-\pi, \pi]$. Note that the $y$-axis ranges are different in top and bottom panels. Since we introduce the effect of LIV in data, but not in theory, then, due to this mismatch in data and theory, we obtain additional contribution to the $\Delta \chi^{2}$ on top of the contribution due to the choices of opposite octants in data and theory. It happens for most of the favorable combinations of $\delta^{\text {true }}$ and $\varphi^{\text {true }}$ as can be seen from Fig. 11. But there are some unfavorable combinations of $\delta^{\text {true }}$ and $\varphi^{\text {true }}$ for which the sensitivities get deteriorated substantially and the values of $\Delta \chi^{2}$ go below the SI case. It suggests that if we minimize the $\Delta \chi^{2}$ over the true values of the standard CP phase $\delta$ and the new dynamical CP phase $\varphi_{e \mu / e \tau}$, the resulting $\Delta \chi^{2}$ attains a value much lower than the value that we obtain in the absence of LIV. 


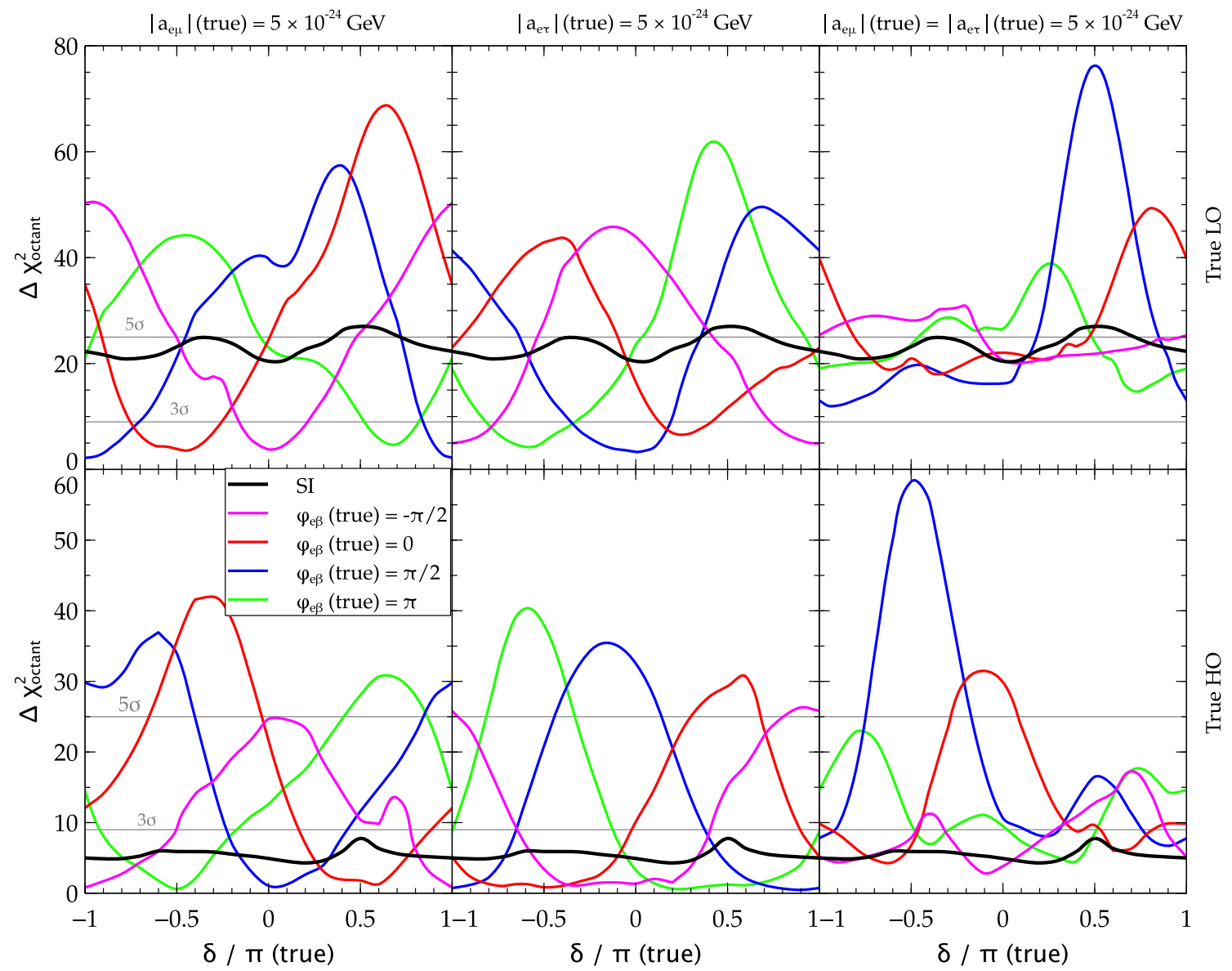

Fig. 11 Octant discovery potential as a function of true $\delta$. In top (bottom) panels, we assume NO-LO (NO-HO) as the true choice with $\theta_{23}^{\text {true }}=42.3^{\circ}\left(47.7^{\circ}\right)$ as benchmark value for LO (HO) case. In all the panels, the data are generated assuming the strength of the true LIV parameter to be $5 \times 10^{-24} \mathrm{GeV}$, and we do not consider the LIV parameters in the fit (theory). The left (middle) panels are for the individual LIV parameter $a_{e \mu}\left(a_{e \tau}\right)$, while the right panels deal with the case when both the LIV parameters are present simultaneously. In each panel, the

\section{References}

1. Particle Data Group Collaboration, M. Tanabashi et al., Review of particle physics. Phys. Rev. D 98(3), 030001 (2018)

2. S. Böser, C. Buck, C. Giunti, J. Lesgourgues, L. Ludhova, S. Mertens, A. Schukraft, M. Wurm, Status of light sterile neutrino searches. arXiv:1906.01739

3. A. Diaz, C.A. Argüelles, G.H. Collin, J.M. Conrad, M.H. Shaevitz, Where are we with light sterile neutrinos? arXiv:1906.00045

4. T2K Collaboration, K. Abe et al., Observation of electron neutrino appearance in a muon neutrino beam. Phys. Rev. Lett. 112, 061802 (2014). arXiv: 1311.4750

5. NOvA Collaboration, D.S. Ayres et al., NOvA: proposal to build a 30 kiloton off-axis detector to study $v_{\mu} \rightarrow v_{e}$ oscillations in the NuMI beamline. arxiv:hep-ex/0503053

6. T2K Collaboration, K. Abe et al., Constraint on the matterantimatter symmetry-violating phase in neutrino oscillations. arXiv: 1910.03887 black curve shows the result for the SI case, while the four colored lines depict the sensitivity for the SI+LIV scheme considering four different true values of $\varphi_{e \mu}$ (left panel), $\varphi_{e \tau}$ (middle panel), and $\varphi_{e \mu}, \varphi_{e \tau}$ (right panel), as mentioned in the legends. We marginalize over $\theta_{23}^{\text {test }}$ in the wrong/opposite octant including the maximal value $\left(45^{\circ}\right)$ and $\delta^{\text {test }}$ in its entire range of $[-\pi, \pi]$. Note that the $y$-axis ranges are different in top and bottom panels
7. NOvA Collaboration, M.A. Acero et al., First measurement of neutrino oscillation parameters using neutrinos and antineutrinos by NOvA. Phys. Rev. Lett. 123(15), 151803 (2019). arXiv:1906.04907

8. P.F. de Salas, D.V. Forero, C.A. Ternes, M. Tortola, J.W.F. Valle, Status of neutrino oscillations: $3 \sigma$ hint for normal mass ordering and improved CP sensitivity. Phys. Lett. B 782(2018), 633-640 (2018). arXiv: 1708.01186

9. Valencia-Globalfit (2018). http://globalfit.astroparticles.es/

10. F. Capozzi, E. Lisi, A. Marrone, A. Palazzo, Current unknowns in the three neutrino framework. Prog. Part. Nucl. Phys. 102, 48-72 (2018). arXiv:1804.09678

11. I. Esteban, M.C. Gonzalez-Garcia, A. Hernandez-Cabezudo, M. Maltoni, T. Schwetz, Global analysis of three-flavour neutrino oscillations: synergies and tensions in the determination of $\theta_{23}, \delta_{C P}$, and the mass ordering. JHEP 01, 106 (2019). arXiv: 1811.05487

12. S. Pascoli, T. Schwetz, Prospects for neutrino oscillation physics. Adv. High Energy Phys. 2013, 503401 (2013) 
13. S.K. Agarwalla, S. Prakash, S. Uma Sankar, Exploring the three flavor effects with future superbeams using liquid argon detectors. JHEP 03, 087 (2014). arXiv: 1304.3251

14. S.K. Agarwalla, Physics potential of long-baseline experiments. Adv. High Energy Phys. 2014, 457803 (2014). arXiv: 1401.4705

15. DUNE Collaboration, R. Acciarri et al., Long-baseline neutrino facility (LBNF) and deep underground neutrino experiment (DUNE). arXiv:1512.06148

16. DUNE Collaboration, R. Acciarri et al., Long-baseline neutrino facility (LBNF) and deep underground neutrino experiment (DUNE). arXiv:1601.02984

17. Hyper-Kamiokande Proto-Collaboration Collaboration, K. Abe et al., Physics potential of a long-baseline neutrino oscillation experiment using a J-PARC neutrino beam and HyperKamiokande. PTEP 2015, 053 C02 (2015). arXiv:1502.05199

18. Hyper-Kamiokande Collaboration, K. Abe et al., Physics potentials with the second Hyper-Kamiokande detector in Korea. PTEP 2018(6), 063C01 (2018). arXiv:1611.06118

19. ESSnuSB Collaboration, E. Baussan et al., A very intense neutrino super beam experiment for leptonic $\mathrm{CP}$ violation discovery based on the European spallation source linac. Nucl. Phys. B 885, 127 149 (2014). arXiv: 1309.7022

20. S.K. Agarwalla, S. Choubey, S. Prakash, Probing neutrino oscillation parameters using high power superbeam from ESS. JHEP 12, 020 (2014). arXiv:1406.2219

21. C.A. Argelles et al., White paper on new opportunities at the nextgeneration neutrino experiments (part 1: BSM neutrino physics and dark matter). arXiv:1907.08311

22. V.A. Kostelecky, M. Mewes, Lorentz and CPT violation in neutrinos. Phys. Rev. D 69, 016005 (2004). arXiv:hep-ph/0309025

23. J.S. Diaz, A. Kostelecky, Lorentz- and CPT-violating models for neutrino oscillations. Phys. Rev. D 85, 016013 (2012). arXiv: 1108.1799

24. O.W. Greenberg, CPT violation implies violation of Lorentz invariance. Phys. Rev. Lett. 89, 231602 (2002). arXiv:hep-ph/0201258

25. V.A. Kostelecky, S. Samuel, Spontaneous breaking of Lorentz symmetry in string theory. Phys. Rev. D 39, 683 (1989)

26. V.A. Kostelecky, S. Samuel, Phenomenological gravitational constraints on strings and higher dimensional theories. Phys. Rev. Lett. 63, 224 (1989)

27. V.A. Kostelecky, R. Potting, CPT and strings. Nucl. Phys. B 359, 545-570 (1991)

28. V.A. Kostelecky, R. Potting, CPT, strings, and meson factories. Phys. Rev. D 51, 3923-3935 (1995). arXiv:hep-ph/9501341

29. V.A. Kostelecky, R. Potting, Expectation values, Lorentz invariance, and CPT in the open bosonic string. Phys. Lett. B 381, 89-96 (1996). arXiv:hep-th/9605088

30. D. Colladay, V.A. Kostelecky, CPT violation and the standard model. Phys. Rev. D 55, 6760-6774 (1997). arXiv:hep-ph/9703464

31. D. Colladay, V.A. Kostelecky, Lorentz violating extension of the standard model. Phys. Rev. D 58, 116002 (1998). arXiv:hep-ph/9809521

32. A. Kostelecky, M. Mewes, Neutrinos with Lorentz-violating operators of arbitrary dimension. Phys. Rev. D 85, 096005 (2012). arXiv: 1112.6395

33. V.A. Kostelecky, M. Mewes, Lorentz violation and shortbaseline neutrino experiments. Phys. Rev. D 70, 076002 (2004). arXiv:hep-ph/0406255

34. T. Katori, V.A. Kostelecky, R. Tayloe, Global three-parameter model for neutrino oscillations using Lorentz violation. Phys. Rev. D 74, 105009 (2006). arXiv:hep-ph/0606154

35. L.S.N.D. Collaboration, L.B. Auerbach et al., Tests of Lorentz violation in anti-nu(mu) -> anti-nu(e) oscillations. Phys. Rev. D 72, 076004 (2005). arXiv:hep-ex/0506067

36. MINOS Collaboration, P. Adamson et al., Testing Lorentz invariance and CPT conservation with NuMI neutrinos in the MINOS near detector. Phys. Rev. Lett. 101, 151601 (2008).arXiv:0806.4945

37. MINOS Collaboration, P. Adamson et al., A search for Lorentz invariance and CPT violation with the MINOS far detector. Phys. Rev. Lett. 105, 151601 (2010). arXiv:1007.2791

38. MINOS Collaboration, P. Adamson et al., Search for Lorentz invariance and CPT violation with muon antineutrinos in the MINOS near detector. Phys. Rev. D 85, 031101 (2012). arXiv: 1201.2631

39. MiniBooNE Collaboration, A.A. Aguilar-Arevalo et al., Test of Lorentz and CPT violation with short baseline neutrino oscillation excesses. Phys. Lett. B 718, 1303-1308 (2013). arXiv:1109.3480

40. Double Chooz Collaboration, Y. Abe et al., First test of lorentz violation with a reactor-based antineutrino experiment. Phys. Rev. D 86, 112009 (2012). arXiv: 1209.5810

41. Super-Kamiokande Collaboration, K. Abe et al., Test of Lorentz invariance with atmospheric neutrinos. Phys. Rev. D 91(5), 052003 (2015). arXiv: 1410.4267

42. IceCube Collaboration, R. Abbasi et al., Search for a Lorentzviolating sidereal signal with atmospheric neutrinos in IceCube. Phys. Rev. D 82, 112003 (2010). arXiv:1010.4096

43. IceCube Collaboration, M.G. Aartsen et al., Neutrino interferometry for high-precision tests of lorentz symmetry with IceCube. Nat. Phys. 14(9), 961-966 (2018). arXiv:1709.03434

44. T2K Collaboration, K. Abe et al., Search for Lorentz and CPT violation using sidereal time dependence of neutrino flavor transitions over a short baseline. Phys. Rev. D 95(11), 111101 (2017). arXiv:1703.01361

45. A. Dighe, S. Ray, CPT violation in long baseline neutrino experiments: a three flavor analysis. Phys. Rev. D 78, 036002 (2008). arXiv:0802.0121

46. G. Barenboim, J.D. Lykken, MINOS and CPT-violating neutrinos. Phys. Rev. D 80, 113008 (2009). arXiv:0908.2993

47. B. Rebel, S. Mufson, The search for neutrino-antineutrino mixing resulting from Lorentz invariance violation using neutrino interactions in MINOS. Astropart. Phys. 48, 78-81 (2013). arXiv: 1301.4684

48. A. de Gouva, K.J. Kelly, Neutrino vs. antineutrino oscillation parameters at DUNE and Hyper-Kamiokande. Phys. Rev. D 96(9), 095018 (2017). arXiv:1709.06090

49. G. Barenboim, C.A. Ternes, M. Tórtola, Neutrinos, DUNE and the world best bound on CPT violation. arXiv: 1712.01714

50. G. Barenboim, M. Masud, C.A. Ternes, M. Trtola, Exploring the intrinsic Lorentz-violating parameters at DUNE. Phys. Lett. B 788, 308-315 (2019). arXiv:1805.11094

51. R. Majhi, C. Soumya, R. Mohanta, Exploring the effect of Lorentz invariance violation in NOvA experiment. arXiv:1907.09145

52. C. Giunti, M. Laveder, Hint of CPT violation in short-baseline electron neutrino disappearance. Phys. Rev. D 82, 113009 (2010). arXiv: 1008.4750

53. A. Datta, R. Gandhi, P. Mehta, S.U. Sankar, Atmospheric neutrinos as a probe of CPT and Lorentz violation. Phys. Lett. B 597, 356361 (2004). arXiv:hep-ph/0312027

54. A. Chatterjee, R. Gandhi, J. Singh, Probing Lorentz and CPT violation in a magnetized iron detector using atmospheric neutrinos. JHEP 06, 045 (2014). arXiv: 1402.6265

55. B. Singh Koranga, P. Khurana, CPT violation in atmospheric neutrino oscillation: a two flavour matter effects. Int. J. Theor. Phys. 53(11), 3737-3743 (2014)

56. J.S. Diaz, T. Schwetz, Limits on CPT violation from solar neutrinos Phys. Rev. D 93(9), 093004 (2016). arXiv:1603.04468

57. D. Hooper, D. Morgan, E. Winstanley, Lorentz and CPT invariance violation in high-energy neutrinos. Phys. Rev. D 72, 065009 (2005). arXiv:hep-ph/0506091

58. G. Tomar, S. Mohanty, S. Pakvasa, Lorentz invariance violation and IceCube neutrino events. JHEP 11, 022 (2015). arXiv:1507.03193 
59. J. Liao, D. Marfatia, IceCube?s astrophysical neutrino energy spectrum from CPT violation. Phys. Rev. D 97(4), 041302 (2018). arXiv: 1711.09266

60. A. Carle, N. Chanon, S. Perries, Prospects for Lorentz Invariance Violation searches with top pair production at the LHC and future hadron colliders. arXiv: 1908.11256

61. N. Chanon, A. Carle, S. Perries, Prospects for Lorentz-violation searches at the LHC and future colliders, in 8th Meeting on CPT and Lorentz Symmetry (CPT'19) Bloomington, Indiana, USA, May 12-16, 2019 (2019). arXiv:1908.11734

62. V.A. Kostelecky, N. Russell, Data tables for Lorentz and CPT violation. Rev. Mod. Phys. 83, 11-31 (2011). arXiv:0801.0287

63. Y. Farzan, M. Tortola, Neutrino oscillations and non-standard interactions. Front. Phys. 6, 10 (2018). arXiv: 1710.09360

64. I. Esteban, M.C. Gonzalez-Garcia, M. Maltoni, On the determination of leptonic $\mathrm{CP}$ violation and neutrino mass ordering in presence of non-standard interactions: present status. JHEP 06, 055 (2019). arXiv: 1905.05203

65. M. Dentler, 1 Hernndez-Cabezudo, J. Kopp, P.A.N. Machado, M. Maltoni, I. Martinez-Soler, T. Schwetz, Updated global analysis of neutrino oscillations in the presence of $\mathrm{eV}$-scale sterile neutrinos. JHEP 08, 010 (2018). arXiv:1803.10661

66. F.J. Escrihuela, D.V. Forero, O.G. Miranda, M. Tortola, J.W.F. Valle, On the description of nonunitary neutrino mixing. Phys. Rev. D 92(5), 053009 (2015). arXiv:1503.08879. [Erratum: Phys. Rev. D 93(11), 119905 (2016)]

67. M. Blennow, P. Coloma, E. Fernandez-Martinez, J. HernandezGarcia, J. Lopez-Pavon, Non-unitarity, sterile neutrinos, and non-standard neutrino interactions. JHEP 04, 153 (2017). arXiv: 1609.08637

68. J.M. Berryman, A. de Gouva, K.J. Kelly, O.L.G. Peres, Z. Tabrizi, Large, extra dimensions at the deep underground neutrino experiment. Phys. Rev. D 94(3), 033006 (2016). arXiv:1603.00018

69. P. Ballett, M. Hostert, S. Pascoli, Y.F. Perez-Gonzalez, Z. Tabrizi, R. Zukanovich Funchal, $Z$ 's in neutrino scattering at DUNE. Phys. Rev. D 100(5), 055012 (2019). arXiv: 1902.08579

70. J.S. Diaz, Correspondence between nonstandard interactions and CPT violation in neutrino oscillations. arXiv: 1506.01936

71. G. Barenboim, C.A. Ternes, M. Tórtola, New physics vs new paradigms: distinguishing CPT violation from NSI. arXiv: 1804.05842

72. T. Kikuchi, H. Minakata, S. Uchinami, Perturbation theory of neutrino oscillation with nonstandard neutrino interactions. JHEP $\mathbf{0 3}$, 114 (2009). arXiv:0809.3312

73. S.K. Agarwalla, S.S. Chatterjee, A. Palazzo, Degeneracy between $\theta_{23}$ octant and neutrino non-standard interactions at DUNE. Phys. Lett. B 762, 64-71 (2016). arXiv:1607.01745

74. M. Masud, S. Roy, P. Mehta, Correlations and degeneracies among the NSI parameters with tunable beams at DUNE. Phys. Rev. D 99(11), 115032 (2019). arXiv:1812.10290
75. B. Roe, Matter density versus distance for the neutrino beam from Fermilab to Lead, South Dakota, and comparison of oscillations with variable and constant density. Phys. Rev. D 95(11), 113004 (2017). arXiv:1707.02322

76. DUNE Collaboration, R. Acciarri et al., Long-Baseline Neutrino Facility (LBNF) and Deep Underground Neutrino Experiment (DUNE) Conceptual Design Report Volume 1: The LBNF and DUNE Projects. arXiv:1601.05471

77. DUNE Collaboration, B. Abi et al., The DUNE far detector interim design report volume 1: physics, technology and strategies. arXiv: 1807.10334

78. P. Huber, M. Lindner, W. Winter, Simulation of long-baseline neutrino oscillation experiments with GLoBES (General Long Baseline Experiment Simulator). Comput. Phys. Commun. 167, 195 (2005). arXiv:hep-ph/0407333

79. P. Huber, J. Kopp, M. Lindner, M. Rolinec, W. Winter, New features in the simulation of neutrino oscillation experiments with GLoBES 3.0: General Long Baseline Experiment Simulator. Comput. Phys. Commun. 177, 432-438 (2007). arXiv:hep-ph/0701187

80. DUNE Collaboration, T. Alion et al., Experiment simulation configurations used in DUNE CDR. arXiv:1606.09550

81. J. Kopp, Efficient numerical diagonalization of hermitian 3 x 3 matrices. Int. J. Mod. Phys. C 19, 523-548 (2008). arXiv:physics/0610206

82. J. Kopp, M. Lindner, T. Ota, J. Sato, Non-standard neutrino interactions in reactor and superbeam experiments. Phys. Rev. D 77, 013007 (2008). arXiv:0708.0152

83. GEANT4 Collaboration, S. Agostinelli et al., GEANT4: a simulation toolkit. Nucl. Instrum. Methods A 506, 250-303 (2003)

84. J. Allison et al., Geant 4 developments and applications. IEEE Trans. Nucl. Sci. 53, 270 (2006)

85. A.M. Dziewonski, D.L. Anderson, Preliminary reference earth model. Phys. Earth Planet. Interiors 25, 297-356 (1981)

86. P. Huber, M. Lindner, W. Winter, Superbeams versus neutrino factories. Nucl. Phys. B 645, 3-48 (2002). arXiv:hep-ph/0204352

87. G.L. Fogli, E. Lisi, A. Marrone, D. Montanino, A. Palazzo, Getting the most from the statistical analysis of solar neutrino oscillations. Phys. Rev. D 66, 053010 (2002). arXiv:hep-ph/0206162

88. M.C. Gonzalez-Garcia, M. Maltoni, Atmospheric neutrino oscillations and new physics. Phys. Rev. D 70, 033010 (2004). arXiv:hep-ph/0404085

89. R. Gandhi, P. Ghoshal, S. Goswami, P. Mehta, S.U. Sankar, S. Shalgar, Mass hierarchy determination via future atmospheric neutrino detectors. Phys. Rev. D 76, 073012 (2007). arXiv:0707.1723

90. X. Qian, A. Tan, W. Wang, J.J. Ling, R.D. McKeown, C. Zhang, Statistical evaluation of experimental determinations of neutrino mass hierarchy. Phys. Rev. D 86, 113011 (2012). arXiv:1210.3651 